\title{
Functional interaction between macrophages and hepatocytes dictate the outcome of liver fibrosis
}

\author{
Min Xie ${ }^{1, \star}\left(\mathbb{D}\right.$, Ren Hui Chia ${ }^{1,3, \star}$, Dan Li $^{1}$, Fanny Xueting Teo ${ }^{1}$, Christian Krueger ${ }^{1}$, Kanaga Sabapathy ${ }^{1,2,3,4}$ (1)
}

\begin{abstract}
Hepatocytes and liver-resident macrophages known as Kupffer cells (KCS) are key cell types involved in liver fibrosis. The transcription factor c-Jun plays a fundamental role in regulating hepatocyte and macrophage functions. We have examined c-Jun's role in the functional interaction of these cells during liver fibrosis induced by carbon tetrachloride. While hepatocytespecific c-jun deletion led to increased fibrosis, the opposite outcome was observed when c-jun was deleted in both hepatocytes and KCs. Molecular analyses revealed compromised cytokine gene expression as the apical event related to the phenotype. Yet, purified hepatocytes from both mouse cohorts showed similar defects in cytokine gene expression. However, we noted increased macrophage infiltration in the absence of c-Jun in hepatocytes, which when chemically depleted, reversed the phenotype. Consistently, $c$-jun deletion in KCs alone also led to reduced fibrosis and cytokine gene expression. By contrast, $c$-jun deletion in hepatocytes and KCs did not affect the resolution phase after fibrotic injury. These data together demonstrate a pro-fibrogenic role for c-Jun in hepatocytes and KCs that functionally interact to regulate liver fibrosis.
\end{abstract}

DOI 10.26508/lsa.202000803 | Received 3 June 2020 | Revised 7 January 2021 | Accepted 15 January 2021 | Published online 29 January 2021

\section{Introduction}

Fibrosis of the liver is a process of wound healing that is activated upon injury to the liver. It occurs under a variety of contexts including viral infection, alcohol overconsumption and fatty liver disease arising from high-fat diet (Trautwein et al, 2015; Koyama \& Brenner, 2017). Chronic stimulation exacerbates the wounding process, leading to severe scarring. This is often seen in the lead-up to the formation of hepatocellular carcinoma (HCC), where stimulation with alcohol or high-fat diet could lead from fibrosis, to cirrhosis and consequently, HCC formation (Michelotti et al, 2013; Koyama \& Brenner, 2017). However, when the stimuli are removed, the scars are resolved by proteases (Ramachandran et al, 2015). Several cell types are involved in orchestrating liver fibrosis. These include the hepatocytes and liver-resident macrophages known also as Kupffer cells (KCS) that produce fibrogenic cytokines to initiate the fibrotic process of activating the hepatic stellate cells (HSCS). Activated HSCs then produce collagens which are deposited as scars. All three cell types contribute to the secretion of chemokines such as CCL2 that leads to the recruitment of bone-marrowderived monocytes, which develop into $L y-6 C^{+}$macrophages that further promote liver injury, leading to further HSC activation (Ju \& Tacke, 2016). Depletion of KCs by several means has been shown to attenuate liver fibrosis (Guillot \& Tacke, 2019), suggesting a critical role for this cell type in liver pathogenesis.

Many signaling pathways have been implicated in regulating liver fibrosis, including hedgehog, JNK, and IKK $\beta$, amongst others (Sun \& Karin, 2008; Seki et al, 2012; Machado \& Diehl, 2018). Of these, the JNKs have been shown to regulate liver fibrosis varyingly. Global JNK1 deficiency or absence of JNK1 in hepatocytes alone have been shown to differentially affect fibrosis induced by carbon tetrachloride $\left(\mathrm{CCl}_{4}\right)$, by choline-deficient diet treatment and by bile-duct ligation (Kodama et al, 2009; Zhao et al, 2014; Cubero et al, 2016). In the case of global JNK1 deficiency, bone marrow-derived cells were thought to be contributory in one case as hepatocyte-specific deletion of JNK1 did not significantly affect fibrosis in that study (Kodama et al, 2009), whereas HSCs rather than bone-marrow derived cells were thought to be causal in another study (Zhao et al, 2014). Moreover, hepatocyte-specific JNK1 deficiency was found not to significantly affect fibrosis (Zhao et al, 2014; Cubero et al, 2016), despite higher levels of liver injury as determined by elevated aspartate aminotransferase (AST) levels. These studies together highlight that the JNK signaling pathway regulates liver fibrosis through multiple cell types in the liver likely through complex interactions.

c-Jun is an integral member of the activator-protein (AP)-1 family of transcription factors and a direct substrate of the JNKs, mediating many of the stress-regulated responses (Eferl \& Wagner, 2003; Shaulian, 2010). It regulates a variety of cellular processes such as

${ }^{1}$ Division of Cellular and Molecular Research, Humphrey Oei Institute of Cancer Research, National Cancer Centre Singapore, Singapore, Singapore ${ }^{2}$ Cancer and Stem Cell
Biology Program, Duke-NUS Medical School, Singapore, Singapore ${ }^{3}$ Department of Biochemistry, Yong Loo Lin School of Medicine, National University of Singapore, Biology Program, Duke-NUS Medical School, Singapore, Singapore ${ }^{3}$ Department of Biochen
Singapore, Singapore ${ }^{4}$ Institute of Molecular and Cellular Biology, Singapore, Singapore

Correspondence: cmrksb@nccs.com.sg

Min Xie's present address is Center for Discovery and Innovation, Hackensack Meridian Health, Nutley, NJ, USA

*Min Xie and Ren Hui Chia contributed equally to this work 
apoptosis, proliferation and differentiation, thereby regulating organismal physiology (Eferl \& Wagner, 2003; Shaulian, 2010). c-Jun is a critical regulator of liver functions in various contexts: it is required for embryonic liver development (Hilberg et al, 1993), is a limiting factor for hepatocellular carcinoma (HCC) formation induced by chemicals (Eferl et al, 2003), and is necessary for liver regeneration upon partial hepatectomy (Behrens et al, 2002). c-Jun expression is up-regulated in many liver conditions such as fibrosis and non-alcoholic steatohepatitis (NASH), both in hepatocytes and non-parenchymal cells (Schulien et al, 2019). A recent study showed that hepatocyte-specific deletion of c-Jun led to increased fibrosis induced by methionine/choline-deficient diet, whereas combined deletion in non-parenchymal cells ameliorated the response (Schulien et al, 2019). Independently, c-Jun has been deleted in macrophages which affected the pro-inflammatory response, reducing bone-destruction in an arthritis model (Hannemann et al, 2017). We have, therefore, investigated the role of c-Jun in hepatocytes and KCs in regulating liver fibrosis. Our results suggest that c-Jun promotes fibrosis through both these cell types, but in a coordinated manner.

\section{Results}

\section{c-jun deletion in hepatocytes alone or together with KCs result in differential effects on liver fibrosis}

Experimental induction of liver fibrosis in mice by repeated $\mathrm{CCl}_{4}$ treatment leads to liver injury (Moles et al, 2014), assessable by Sirius red staining that marks the deposition of collagen in the fibrotic area, reflecting the extent of injury (Fig S1A). Continual $\mathrm{CCl}_{4}$ treatment was accompanied by a significant increase in the expression of c-jun in the liver (Fig S1B). Consistently and similar to published observations in humans (Schulien et al, 2019), c-Jun levels were also up-regulated in the liver (Fig S1C), concomitant to an increase in the expression of phosphorylated-JNK (Fig S1D), indicative of a plausible role for the JNK-c-Jun pathway in regulating the hepatic fibrosis process.

Given the extensive analysis of the role of JNKs in liver fibrosis (Seki et al, 2012), we undertook a genetic approach by deleting c-Jun in hepatocytes and KCs to understand its role in the liver fibrotic process. We used the c-Jun floxxed $\left(\mathrm{c}-\mathrm{jun}{ }^{\mathrm{f} / \mathrm{f}}\right)$ mouse strain in which the c-jun gene is flanked by loxP sites (Behrens et al, 2002) (Fig S2A), and crossed them to a variety of mouse strains expressing the Crerecombinase under the following promoters: Mx1-cre (for deletion mainly in hepatocytes and hematopoietic cells including monocytes) (referred hereafter as Mx-cre), albumin-cre (for deletion in hepatocytes only), and lysozyme-M (LysM)-cre (monocyte-specific deletion, including KCS) (Lanaya et al, 2014) (Fig 1A). Poly-I:C or tamoxifen were injected to induce the expression of the crerecombinase in the $\mathrm{Mx}$-cre or to activate the creER-recombinase in Alb-cre strains, respectively, along with control mice. Liver fibrosis was induced by bi-weekly $\mathrm{CCl}_{4}$ injections, as shown in the work-flow and treatment protocol diagram (Fig 1B), and all mice were euthanized after 8 wk of treatment for detailed analyses. Evaluation of deletion of c-jun using whole livers at euthanasia showed extensive $c$-jun deletion in the different groups of mice (Fig $\mathrm{S} 2 \mathrm{~B})$. We further purified hepatocytes and $\mathrm{KCS}$ from the respective cohorts of mice, which again showed specific and significant de-

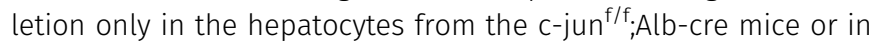
both the hepatocytes and $\mathrm{KCs}$ in livers from the $\mathrm{C}$-jun ${ }^{\mathrm{f} / \mathrm{f}} ; \mathrm{Mx}$-cre mice (Fig S2C).

c-Jun expression was significantly reduced in livers of c-jun ${ }^{\mathrm{f} / \mathrm{f}}$; Mx-cre and c-jun ${ }^{\mathrm{f} / \mathrm{f}} ; \mathrm{Alb}$-cre mice at euthanasia (Fig 1C), highlighting the impact of its deletion in hepatocytes that account for more than $80 \%$ of the cellular population in the liver, as previously reported (Behrens et al, 2002). It is noteworthy that Alb-cre-mediated c-jun deletion resulted in slightly higher residual levels of c-Jun in the livers than that of Mx-cre-mediated deletion ( $~ 80 \%$ and $97 \%$ decrease in c-Jun expression in livers of c-jun ${ }^{\mathrm{f} / \mathrm{f}} ; \mathrm{Alb}$-cre and $\mathrm{c}-\mathrm{jun} \mathrm{n}^{\mathrm{f} / \mathrm{f}}$; $M x$-cre, respectively), likely reflecting the expansive deletion beyond hepatocytes in the latter case.

Sirius red staining revealed a significant increase in fibrosis in the $\mathrm{c}$-jun ${ }^{\mathrm{f} / \mathrm{f}} ; \mathrm{Alb}$-cre mice (\% Sirius red staining of $\mathrm{CCl}_{4}$-treated livers: without or with Alb-cre $-6.003 \pm 1.958$ versus $18.614 \pm 10.407$; $P=$ 0.009) (Fig 1D, left). However and strikingly, c-jun deletion in the $\mathrm{c}$-jun ${ }^{\mathrm{f} / \mathrm{f}}$;Mx-cre mice led to a consistent decrease in fibrosis (\% Sirius red staining of $\mathrm{CCl}_{4}$-treated livers: without or with $\mathrm{Mx}$-cre $-4.698 \pm$ 0.709 versus $4.018 \pm 0.476 ; P=0.033$ ) (Fig $1 \mathrm{D}$, right), indicating that c-jun deletion in hepatocytes alone or in combination with KCs result in differential outcomes. A similar trend was observed with alanine aminotransferase (ALT) and aspartate aminotransferase (AST) levels (Fig 1E). These results together suggest a causal role for c-Jun in regulating liver fibrosis, likely through interplay between the hepatocytes and KCs.

\section{Differential effects on cytokine gene expression upon c-jun deletion in hepatocytes alone or in combination with KCs}

To understand the molecular basis for the observed differential effects of c-jun deletion in hepatocytes alone or in combination with $\mathrm{KCS}$, we analyzed the expression of selected genes from three groups corresponding to various processes that coordinately regulate liver fibrosis: (i) Acta2 ( $\alpha-S M A)$, Desmin and Vimentin, whose elevated expression reflects the activation status of the HSCs; (ii) Tgf- $\beta 1, T n f-\alpha$, and Pdgf- $\beta$, the inflammatory cytokines that are produced by hepatocytes and $\mathrm{KCS}$ to induce the fibrotic re-

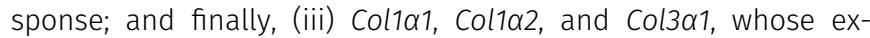
pression is induced for collagen synthesis and deposition by the activated HSCs (Fig 2A). Quantitative real-time (qRT) PCR analysis revealed that HSC activation, as measured by Acta2, Desmin, and Vimentin expression, was compromised in $\mathrm{C}-\mathrm{jun}^{\mathrm{f} / \mathrm{f}} ; \mathrm{MX}$-cre mice, corroborating with the observed reduction in fibrosis (relative expression of Acta2 in livers of $\mathrm{CCl}_{4}$-treated mice: without or with Mx-cre $-4.931 \pm 2.816$ versus $2.523 \pm 1.341, P=0.0277$; Desmin $-3.182 \pm$ 1.028 versus $2.096 \pm 0.634, P=0.0088$; Vimentin $-4.319 \pm 1.780$ versus $2.528 \pm 0.840, P=0.0111$ ) (Fig 2B). On the contrary, and consistent with the observed increased fibrosis, there was a tendency for enhanced HSC activation in the $\mathrm{c}$-jun ${ }^{\mathrm{f} / \mathrm{f}} ; \mathrm{Alb}$-cre mice (relative expression of Acta2 in livers of $\mathrm{CCl}_{4}$-treated mice: without or with Alb-cre $-5.679 \pm$ 0.7799 versus $6.912 \pm 0.7711, P=0.0412$; Desmin $-1.479 \pm 0.7008$ versus $2.476 \pm 0.3532, P=0.0293$ ) (Fig 2C). 
A

B

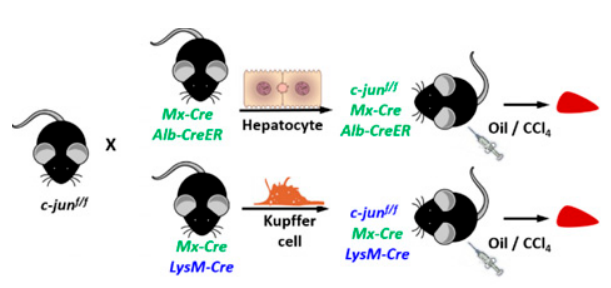

B
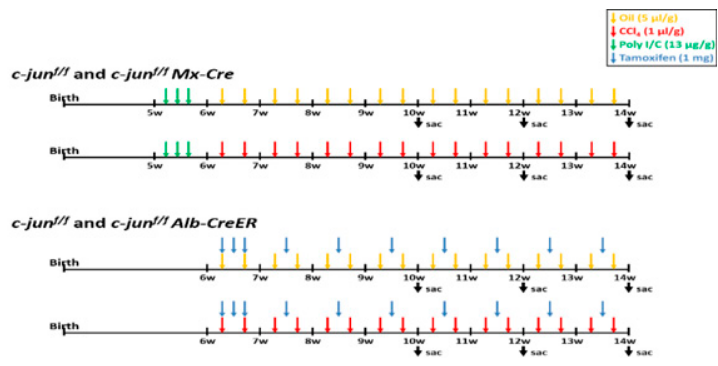

\section{C}
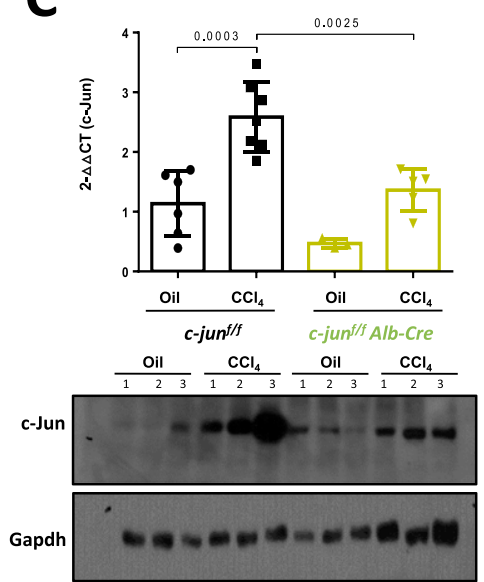
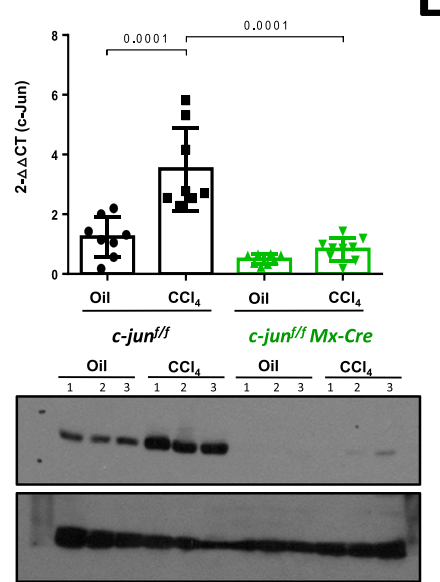

D
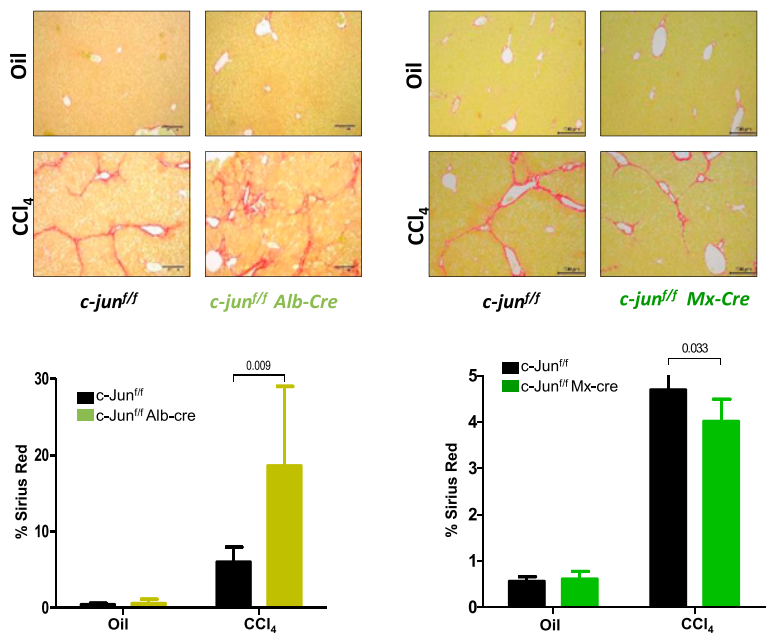

E
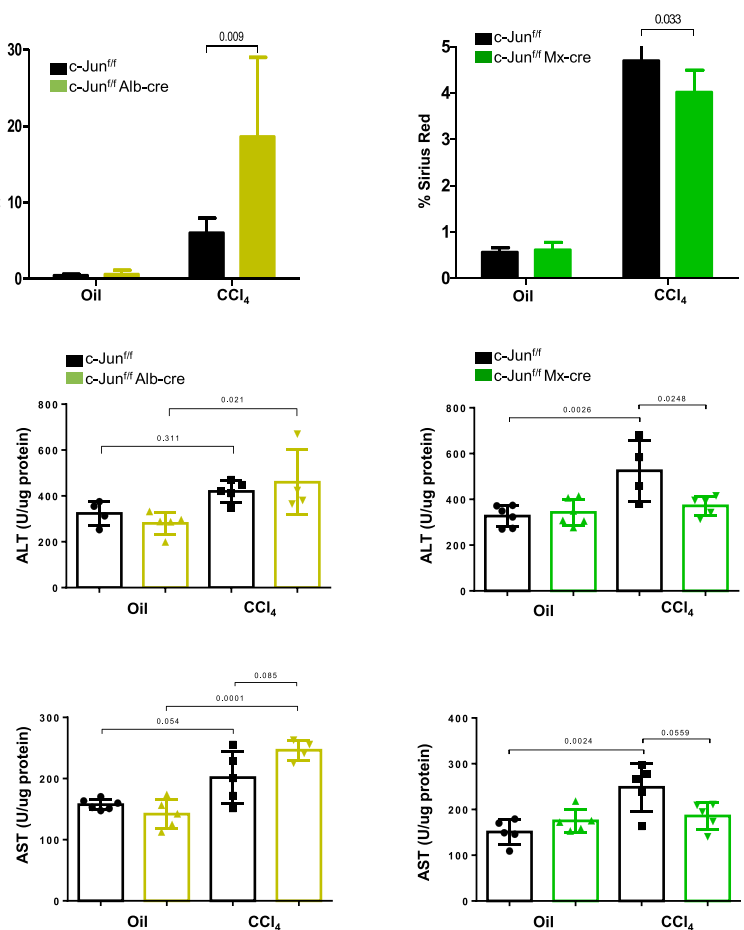

Figure 1. c-jun deletion in hepatocytes alone or together with Kupffer cells results in opposite effects on fibrosis

$(A, B)$ Breeding scheme of mouse strains used in the study. (A) $c$-jun $n^{f / f}$ mice were crossed to the indicated strains of Cre-expressing mice to delete $c$-jun in the various cell types (A). (B) Mice were either treated with oil or carbon tetrachloride $\left(\mathrm{CCl}_{4}\right)$ twice per week for 8 wk to induce fibrosis, and livers were harvested at $72 \mathrm{~h}$ after the last injection (B). Poly l:C or tamoxifen was injected as indicated to induce the expression of Cre in Mx-cre or to activate the Cre-ER in Alb-cre mice, respectively. (C) Expression of c-jun was determined by quantitative real-time PCR (upper panel) and by immunoblotting (lower panel) using whole livers from the indicated mice. Experiments were performed in duplicates and

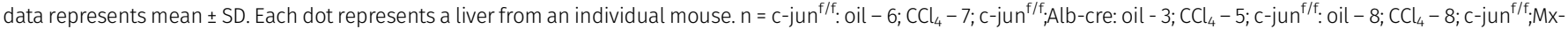
cre: oil - 8; $\mathrm{CCl}_{4}-9$. Each number represents a mouse in the immunoblot analysis. Statistics was performed by two-way ANOVA. Multiple blots were run with the same lysates for detection with the various antibodies. The Gapdh blot was performed once for all lysates, and the blot shown here is the same as shown in Figs $2 \mathrm{~F}$ and S1D left panel, as the same set of lysates was used in these figures. (D, E) Extent of liver fibrosis and damage after 8 wk of oil or $\mathrm{CCl}_{4}$ injection was determined by Sirius red staining (top panels), and representative pictures are shown (10× magnification) (D). Quantification of fibrosis is based on analyzing 20 randomly chosen fields from four individual liver lobes per mouse

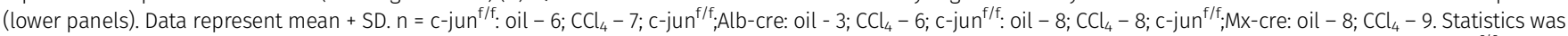

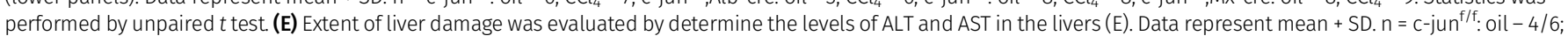

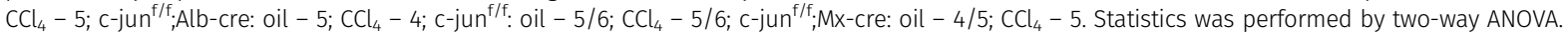

Source data are available for this figure.

We, therefore, evaluated if the induction of the fibrotic response contributed to the deregulated effects observed on HSCS. Analyses of a few cytokines gene expression revealed a defect in several of them, including Tgf- $\beta 1$, Tnf- $\alpha$ and $P d g f-\beta$ in $\mathrm{c}-j u n^{\mathrm{f} / \mathrm{f}}$;Mx-cre mice (relative expression of Tgfb1 in livers of $\mathrm{CCl}_{4}$-treated mice: without or with $\mathrm{Mx}$-cre $2.422 \pm 0.6773$ versus $1.663 \pm 0.5785, P=0.0189 ;$ Tnf $-\alpha-2.539 \pm 1.080$ versus $1.786 \pm 0.7205, P=0.007 ; P d g f-\beta-2.958 \pm 0.9613$ versus $1.843 \pm 0.9280, P=$ 0.0135 ) (Fig 2D), but an opposite effect was generally noted in $\mathrm{C}-\mathrm{jun}{ }^{\mathrm{f} / \mathrm{f}} ; \mathrm{Alb}-$ cre mice (relative expression of $\mathrm{Tnf}-\alpha$ in livers of $\mathrm{CCl}_{4}$-treated mice: without or with Alb-cre $-1.620 \pm 0.8758$ versus $5.385 \pm 1.143, P=0.0324$;
Pdgf $-\beta-2.640 \pm 1.686$ versus $7.629 \pm 2.604, P=0.0005)$ (Fig 2E), suggesting that the differential effects observed on HSC activation may stem from the alterations in the initial process of fibrosis induction. Consistent with the qRT-PCR data, expression of Acta2, Desmin, Vimentin, TNF- $\alpha$ and TGF-

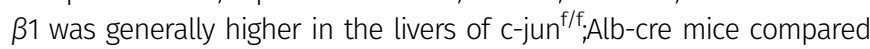

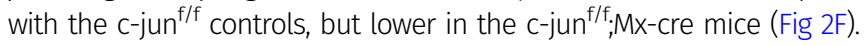

Furthermore and consistent with the altered HSC activation, production of collagen synthesis was also compromised in the livers of the $\mathrm{C}^{\mathrm{Cjun}}{ }^{\mathrm{f} / \mathrm{f}} ; \mathrm{Mx}$-cre mice (relative expression of Col1a1 in livers of $\mathrm{CCl}_{4}$-treated mice: without or with $M x$-cre $-27.71 \pm 17.93$ versus $21.64 \pm 11.07, P=0.6616$; 

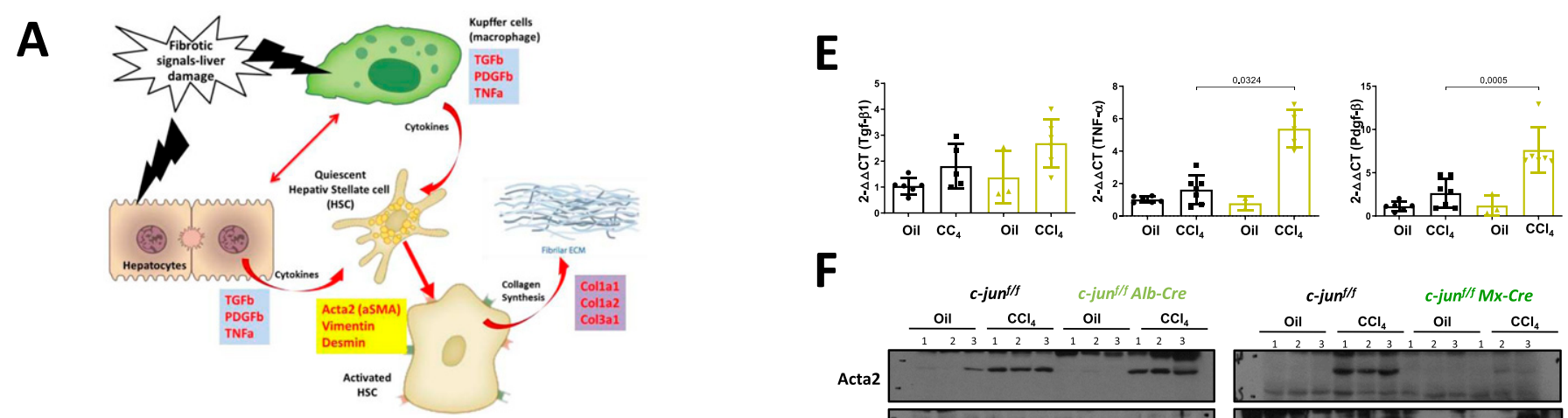

B
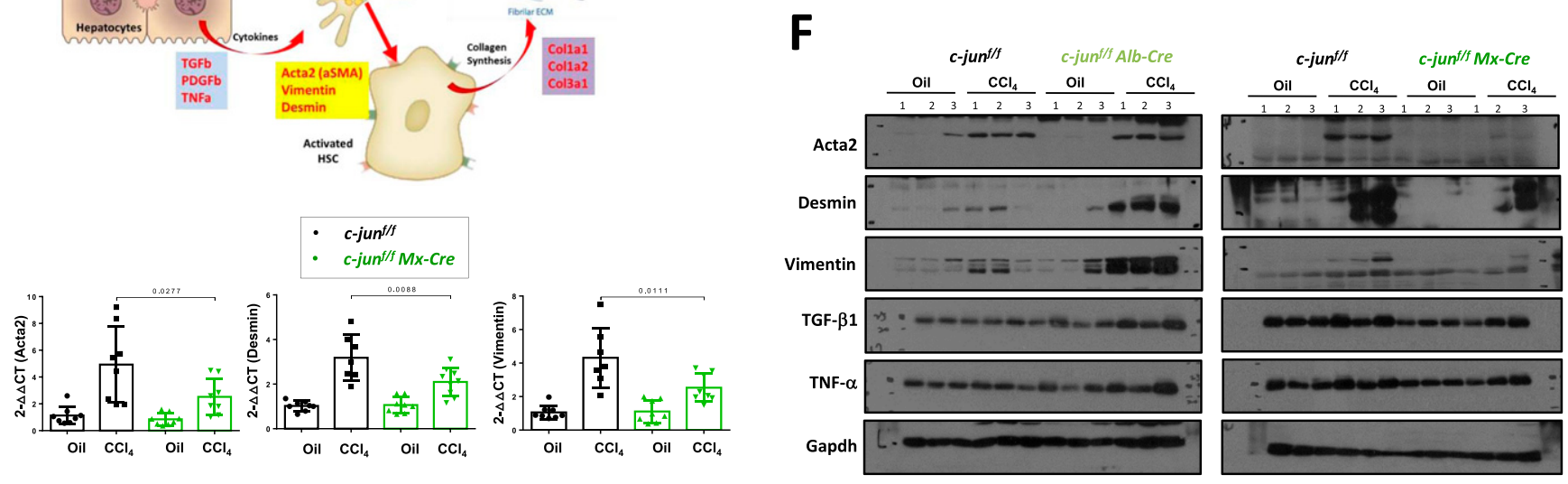

C

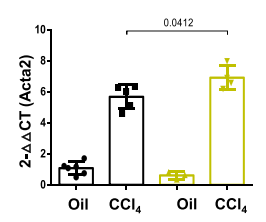

D

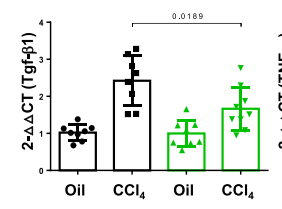

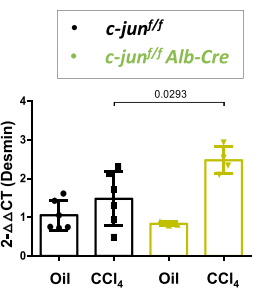

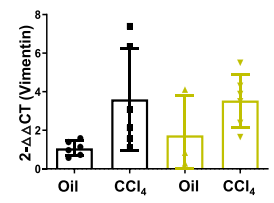

G
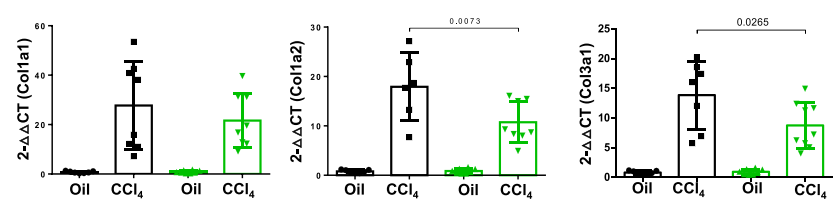

H

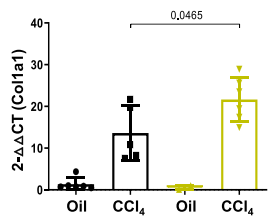

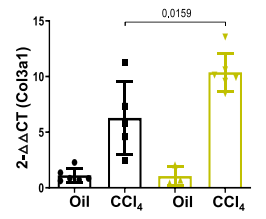

Figure 2. c-jun deletion in hepatocytes alone or together with Kupffer cells affects cytokine gene expression differentially.

(A) Schematic of genes analyzed by quantitative real-time (qRT)-PCR to determine the role of c-Jun in the liver. The three groups of genes analyzed include cytokines produced by hepatocytes and Kupffer cells, hepatic stellate cell activation markers and fibrogenic procollagen genes. (B, C) qRT-PCR analysis was performed using livers

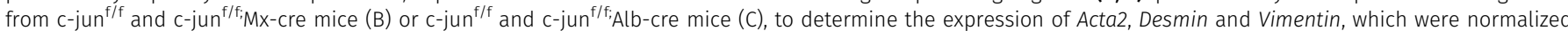
against Gapdh. Experiments were performed in duplicates, and data represent mean $\pm \mathrm{SD}$. Each dot represents a liver from each mouse. $\mathrm{n}=\mathrm{C}-\mathrm{jun}{ }^{\mathrm{f} / \mathrm{f}}$ : oil -6 ; $\mathrm{CCl} \mathrm{C}_{4}-5 / 6$;

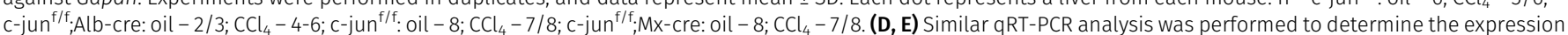
of Tgf- $\beta 1$, Tnf- $\alpha$ and $P d g f-\beta$, which were normalized against Gapdh. Experiments were performed in duplicates, and data represent mean \pm SD. Each dot represents a liver

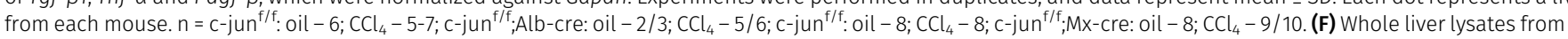
the indicated groups of mice were used for immunoblotting analysis with the indicated antibodies. The Gapdh blot was performed once for all lysates, and the blot shown here is the same as shown in Figs 1C and S1D left panel, as the same set of lysates was used in these figures. (G, H) qRT-PCR analysis was performed as described above to determine the expression of Col1 11 , Col1 $\alpha 2$ and Col3 $\alpha 1$, which were normalized against Gapdh. Experiments were performed in duplicates, and data represent mean \pm

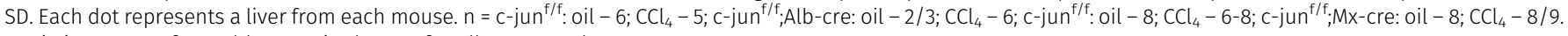
Statistics was performed by unpaired $t$ test for all qRT-PCR data.

Source data are available for this figure.

Col1a2 $-17.91 \pm 6.877$ versus $10.74 \pm 4.176, P=0.0073$; Col3a1 $-13.87 \pm 5.735$ versus $8.751 \pm 3.871, P=0.0265)$, but generally increased in the $\mathrm{c}-\mathrm{jun}{ }^{\mathrm{f} / \mathrm{f}} ; \mathrm{Alb}-$ cre mice (relative expression of Col1a1 in livers of $\mathrm{CCl}_{4}$-treated mice: without or with Alb-cre $-13.62 \pm 6.634$ versus $21.66 \pm 5.286, P=0.0465$; Col1a2 $-6.897 \pm 3.891$ versus $10.24 \pm 1.427, P=0.0710$; Col3a1 $-6.285 \pm 3.303$ versus $10.38 \pm 1.713, P=0.0159$ ) (Fig $2 \mathrm{G}$ and $\mathrm{H}$ ). Collectively, these data

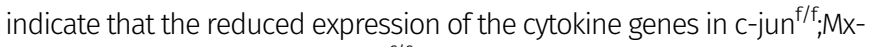
cre mice and vice versa in $c$-jun ${ }^{\mathrm{f} / \mathrm{f}}$ Alb-cre mice is the likely cause of the differential effects observed on HSC activation and fibrosis after c-jun deletion, either in hepatocytes alone or together with KCS.

\section{Non-cell autonomous effects are causal to increased fibrosis upon hepatocyte-specific c-jun deletion}

To understand the observed differential effects, we first evaluated the levels of proliferation (by Ki67 staining) and cell death (by 
TUNEL staining) in the livers of the $\mathrm{CCl}_{4}$-treated $\mathrm{c}$-jun ${ }^{\mathrm{f} / \mathrm{f}} ; \mathrm{Alb}$-cre and

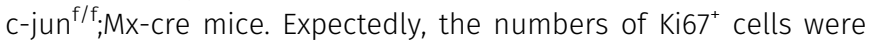
reduced in both the $\mathrm{c}$-jun ${ }^{\mathrm{f} / \mathrm{f}} ; \mathrm{Alb}$-cre mice and $\mathrm{c}$-jun ${ }^{\mathrm{f} / \mathrm{f}} ; \mathrm{Mx}$-cre mice (Fig 3A). This observation is consistent with previous findings that c-Jun is required for the proliferation of hepatocytes upon liver injury (Behrens et al, 2002). There were also no differences noted in cell death at the point of euthanasia in the $\mathrm{c}$-jun ${ }^{\mathrm{f} / \mathrm{f}}$; $\mathrm{MX}$-cre mice (Fig S2D). We, therefore, purified hepatocytes from both groups of mice and evaluated the expression of several cytokine genes which were induced by $\mathrm{CCl}_{4}$ treatment. Interestingly, hepatocytes from both groups of mice in which c-jun was deleted (Fig S2C) had a significant and similar reduction in the induction of these cytokine genes (Fig $3 \mathrm{~B})$, suggesting that the observed differential effects on fibrosis may not be attributable to the differential effects of c-jun deletion in hepatocytes in these two cases.

We, therefore, hypothesized that there may be compensatory or counteracting mechanisms in place to mitigate the lack of hepatocyte function in the $\mathrm{c}$-jun $^{\mathrm{f} / \mathrm{f}}$;Alb-cre cohorts, leading to the observed enhanced fibrosis. Although immunohistochemical analysis showed a significant reduction of c-Jun expression in hepatocytes in both groups of mice after $\mathrm{CCl}_{4}$ treatment, we noted that there was an increase in the number of macrophage-like cells infiltrating the

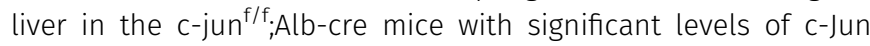
expression (Fig 3C). To confirm the identity of the cells, we stained the sections with $\mathrm{F} 4 / 80$ to detect macrophages, which were enriched in the livers from the $c$-jun ${ }^{\mathrm{f} / \mathrm{f}} ; \mathrm{Alb}$-cre mice (Fig 3D),
A

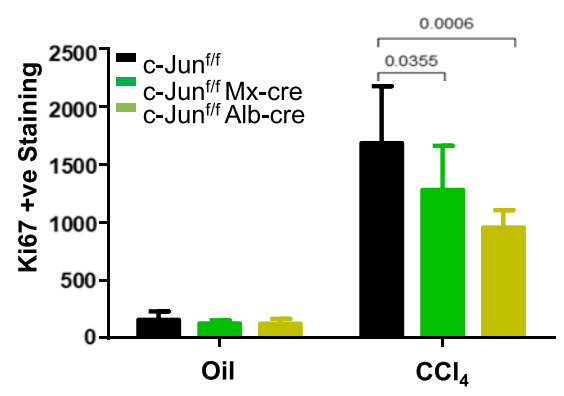

B
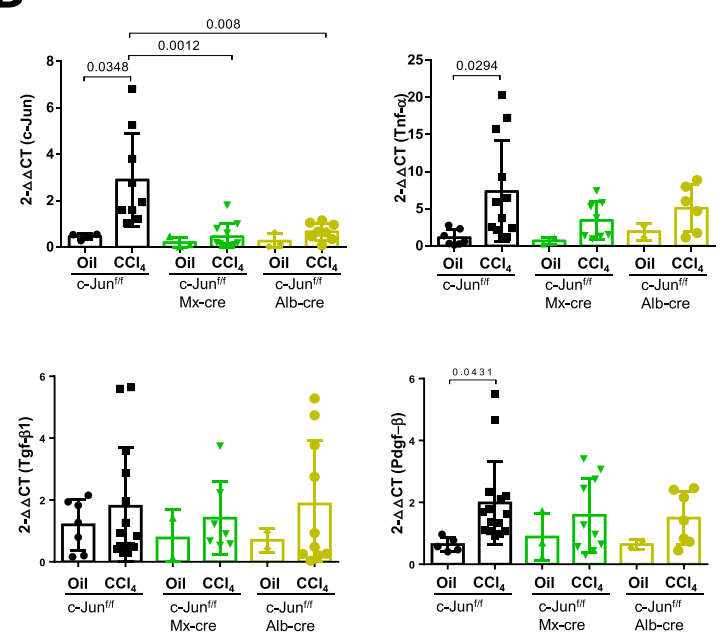

C
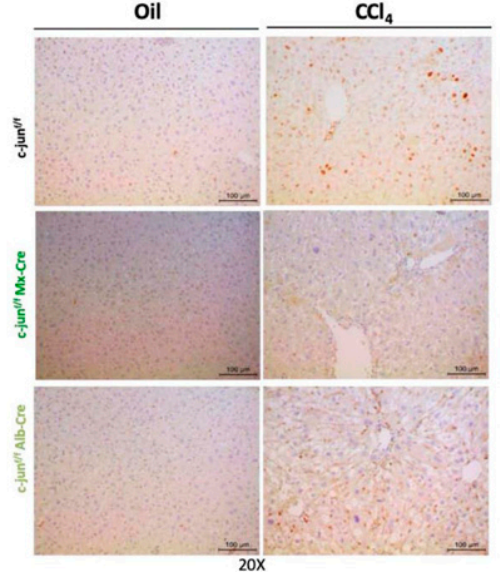

D

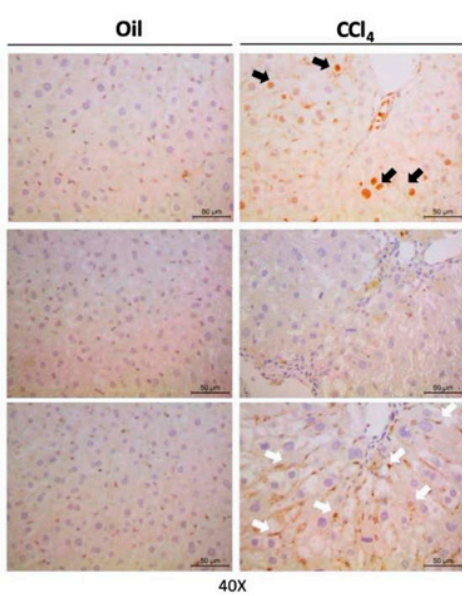

$40 \mathrm{X}$
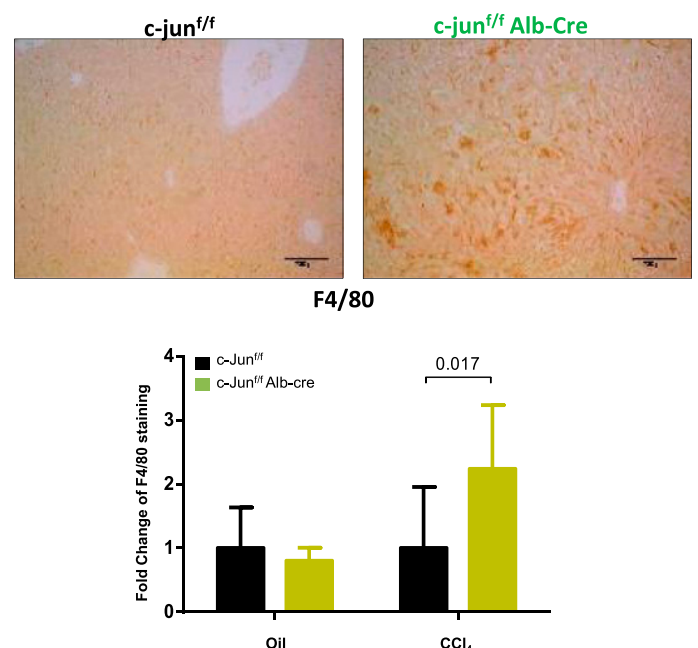

Figure 3. Increase in Kupffer cells in livers of mice with hepatocyte-specific c-jun deletion.

(A) The number of proliferating cells was determined on the liver sections by Ki67 staining. Data represent mean \pm SD. For Ki67 staining, $\mathrm{n}=\mathrm{C}-\mathrm{jun}{ }^{\mathrm{f} / \mathrm{f}}$ : oil -9 ; $\mathrm{CCl} \mathrm{L}_{4}-14$;

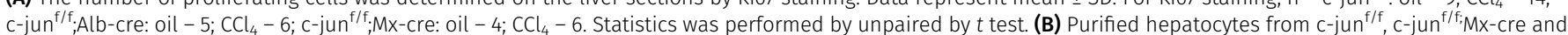

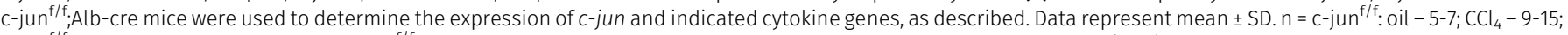

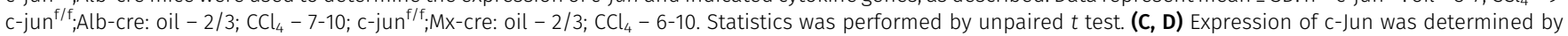
immunohistochemical staining of liver tissues from the indicated mouse strains after 8 wk of $\mathrm{CCl}_{4}$ or oil injection. Representative images are shown. Black arrows indicate the presence of c-Jun positive hepatocytes (reddish brown). (C) White arrows indicate c-Jun positive macrophage-like cells (C). Numbers of F4/80 positive macrophages were quantified (bottom panel) after immunohistochemical staining. (D) Representative pictures are shown (top panel) (D). Data represent mean + SD. C-jun ${ }^{\mathrm{f} / \mathrm{f}}$ : oil - 10; $\mathrm{CCl}_{4}-14 ; \mathrm{c}-\mathrm{jun}{ }^{\mathrm{f} / \mathrm{f}} ; \mathrm{Alb}$-cre: oil - 2; $\mathrm{CCl}_{4}-6$. Statistics was performed by unpaired $t$ test. 
suggesting that elevated levels of KCs and infiltrating macrophages in the absence of c-jun may be causal to the observed enhanced fibrosis in this group of mice.

\section{Monocyte-specific c-jun deletion results in reduced fibrosis}

To investigate the causal role of macrophages to the observed increased fibrosis, we evaluated the effects of depleting macrophages from c-jun ${ }^{\mathrm{f} / \mathrm{f}}$; Alb-cre mice. To this end, $\mathrm{c}$-jun ${ }^{\mathrm{f} / \mathrm{f}}$;Alb-cre mice were treated gadolinium chloride $\left(\mathrm{GdCl}_{3}\right)$, which has been shown to deplete macrophages in vivo (Zhu et al, 2018). $\mathrm{GdCl}_{3}$ treatment led to a significant reduction in c-Jun expression in livers of $\mathrm{CCl}_{4}$-treated $\mathrm{c}$-jun ${ }^{\mathrm{f} / \mathrm{f}}$;Alb-cre mice (Fig S3A), and a concomitant decrease in F4/80 expressing cells (Fig S3B). Analysis of fibrosis by Sirius red staining also showed a reversal and marked reduction of fibrosis in $\mathrm{CCl}_{4}$-treated $\mathrm{c}_{\text {-jun }}{ }^{\mathrm{f} / \mathrm{f}}$;Alb-cre mice upon $\mathrm{GdCl}_{3}$ treatment (\% Sirius red staining of $\mathrm{CCl}_{4}$-treated livers: without or with Alb-cre: for PBS $-5.875 \pm 1.384$ versus $\mathrm{GdCl}_{3}-3.734 \pm 0.341, P=0.0016$; and $\mathrm{PBS}-15.698 \pm 7.898$ versus $\mathrm{GdCl}_{3}-3.502 \pm 1.029, P=0.01$ ) (Fig 4A). Further analysis of expression of multiple genes regulating HSC activation, cytokine production, and collagen synthesis revealed a concomitant reversal of all parameters in $\mathrm{GdCl}_{3}$ treated $\mathrm{c}$-jun ${ }^{\mathrm{f} / \mathrm{f}}$; Alb-cre mice (Fig 4B), implying a compensatory contributory role for KCs to the enhanced liver

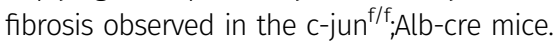

We, therefore, next evaluated if c-jun deletion in $\mathrm{F} 4 / 80^{+}$ monocytic lineage cells alone would also lead to reduced fibrosis. To this end, c-jun was deleted using the LysM-cre mice (Fig S2B), which led to a consistent decrease in $\mathrm{CCl}_{4}$-mediated fibrosis (\% Sirius red staining: without or with LysM-cre $-5.091 \pm 2.143$ versus $3.747 \pm 0.908, P=0.0577$ ) (Fig 5A). Importantly, the cytokine genes expression was significantly impaired in the $\mathrm{c}$-jun ${ }^{\mathrm{f} / \mathrm{f}} ; \mathrm{LysM}$-cre mice (relative expression of Tgfb1 in livers of $\mathrm{CCl}_{4}$-treated mice: without or with LysM-cre $-2.293 \pm 0.3946$ versus $1.742 \pm 0.2081, P=0.0411$; Tnf$\alpha-2.150 \pm 0.8507$ versus $1.114 \pm 0.2720, P=0.0469 ; P d g f-\beta-3.259 \pm$ 1.267 versus $2.037 \pm 0.5995, P=0.045$ ) (Fig 5B). Consistently, HSC activation and collagen synthesis were also compromised by deletion of $c$-jun in the monocytic cells (relative expression of Acta2 in livers of $\mathrm{CCl}_{4}$-treated mice: without or with LysM-cre $-5.771 \pm 2.320$ versus $3.521 \pm 1.026, P=0.0378$; Desmin $-2.065 \pm 0.6768$ versus $1.997 \pm$ $0.6518, P=0.8297$; Vimentin $-3.590 \pm 0.8778$ versus $2.483 \pm 0.6671, P=$ 0.0118; Col1a1 $-44.49 \pm 12.51$ versus $29.24 \pm 12.64, P=0.0335$; Col1a2 $19.81 \pm 6.227$ versus $10.64 \pm 3.899, P=0.0009 ;$ Col3 $\alpha 1-16.64 \pm 5.464$ versus $9.720 \pm 3.472, P=0.0045$ ) (Fig $5 C$ and $D$ ). As noted with the livers of the $\mathrm{c}-\mathrm{jun}{ }^{\mathrm{f} / \mathrm{f}} ; \mathrm{Mx}$-cre mice, the expression of Acta2, Desmin, Vimentin, TNF- $\alpha$ and TGF- $\beta 1$ was significantly reduced in the livers of $\mathrm{c}$-jun ${ }^{\mathrm{f} / \mathrm{f}} ;$ LysM-cre mice compared to the $\mathrm{c}$-jun ${ }^{\mathrm{f} / \mathrm{f}}$ controls (Fig 5E). Collectively, these data indicate that c-Jun is required for the
A
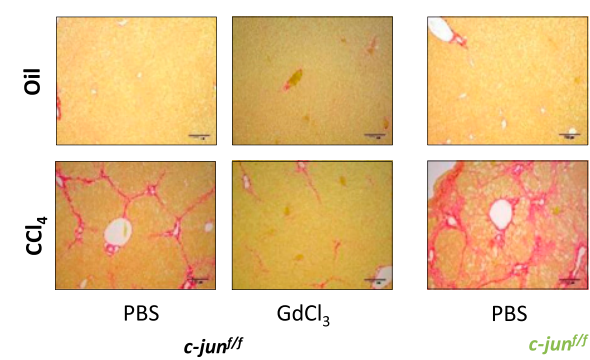
$c-j u n f / /$

c-junf/s Alb-Cre

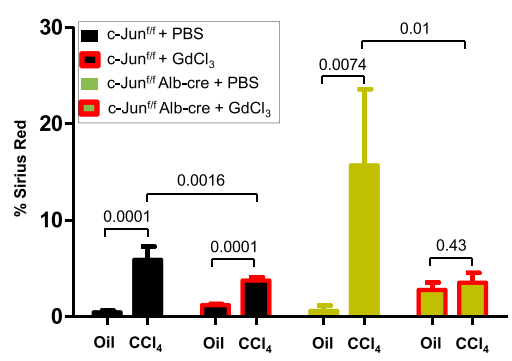

B
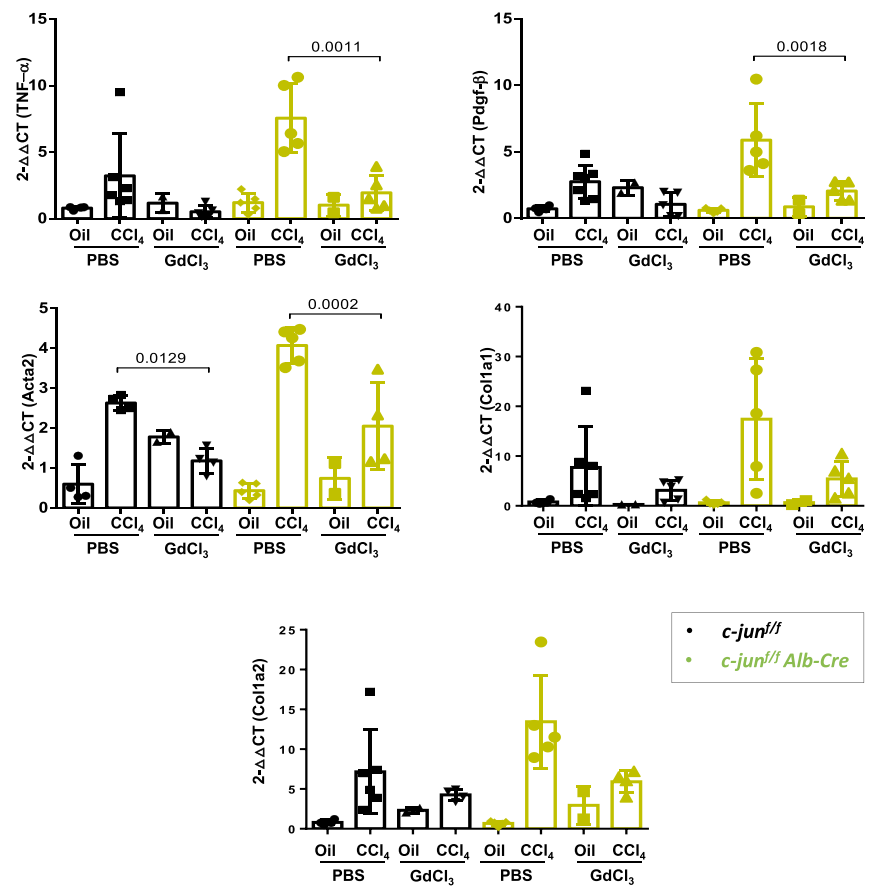

Figure 4. Depletion of macrophages reverses fibrosis in mice with hepatocyte-specific c-jun deletion.

$(\mathbf{A}, \mathbf{B}) \mathrm{c}$-jun ${ }^{\mathrm{f} / \mathrm{f}}$ or c-jun ${ }^{\mathrm{f} / \mathrm{f}} ; \mathrm{Alb}$-cre mice were treated with gadolinium chloride $\left(\mathrm{GdCl}_{3}\right)$ or PBS once a week for 8 wk to deplete the macrophages, concomitant with oil or $\mathrm{CCl}_{4}$ treatment. (A, B) The extent of fibrosis (A), and the expression of the indicated genes from whole livers (B) were then evaluated as described. Experiments were performed in duplicates, and data represent mean $\pm \mathrm{SD}$. (B) Each dot represents a liver from each mouse (B). $\mathrm{n}=\mathrm{C}-\mathrm{jun}{ }^{\mathrm{f} / \mathrm{f}}:$ oil $/ \mathrm{PBS}-4 ; \mathrm{CCl}_{4} / \mathrm{PBS}-4-7 ; \mathrm{oil} / \mathrm{GdCl}_{3}-2 ; \mathrm{CCl}_{4} / \mathrm{GdCl}_{3}-4-6 ; \mathrm{C}-$ jun ${ }^{\mathrm{f} / \mathrm{f}} ; \mathrm{Alb}$-cre: oil/PBS - 5; $\mathrm{CCl}_{4} / \mathrm{PBS}$ - 5; oil/ $\mathrm{GdCl}_{3}-2 ; \mathrm{CCl}_{4} / \mathrm{GdCl}_{3}-4 / 5$. Statistics was performed by two-way ANOVA. 
A

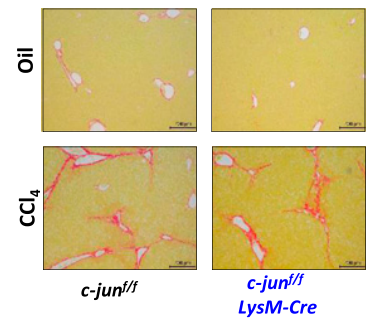

B

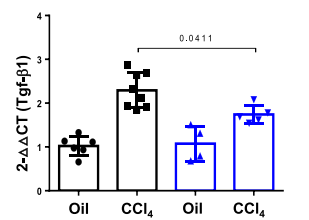

C
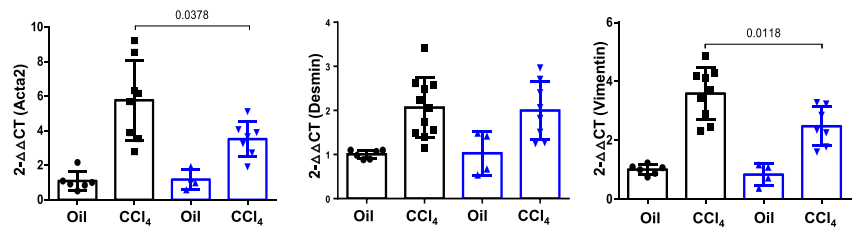

D
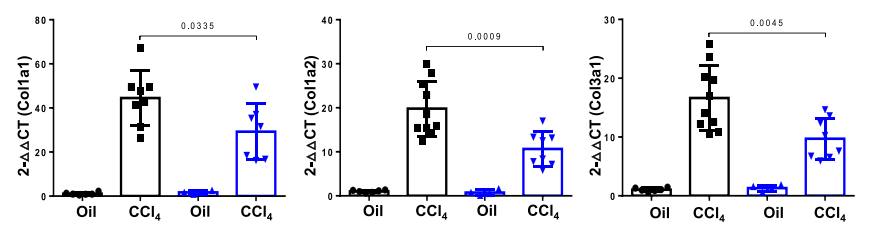

$\mathbf{E}$

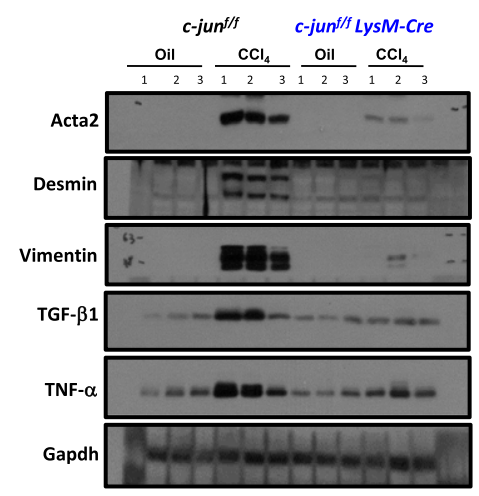

$\mathbf{F}$
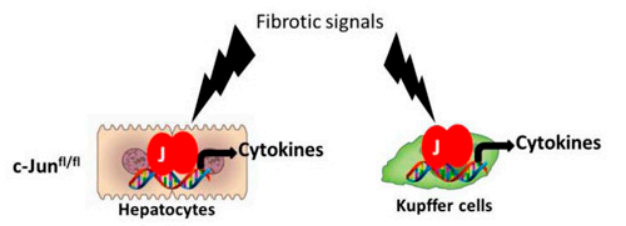

$\underline{\text { Fibrosis }}$

$++$
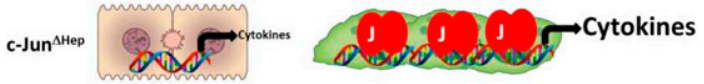

$+++$
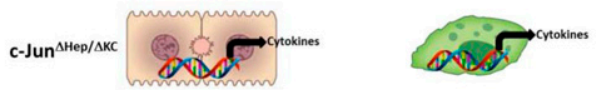

$+$
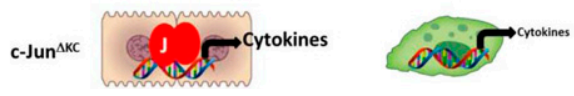

Figure 5. C-Jun is a positive regulator of fibrosis in Kupffer cells (KCs).

(A) Extent of fibrosis was determined by Sirius red staining (left panels), and quantified (right panels) using livers from c-jun ${ }^{\mathrm{f} / \mathrm{f}}$ and $\mathrm{c}$-jun ${ }^{\mathrm{f} / \mathrm{f}}$;LysM-cre mice (A).

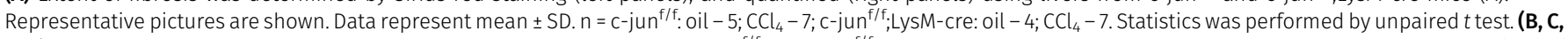
D, E) Quantitative real-time-PCR analysis was performed using livers from c-jun ${ }^{\mathrm{f} / \mathrm{f}}$ and $\mathrm{C}$-jun ${ }^{\mathrm{f} / \mathrm{f}} ;$ LysM-cre mice, to determine the expression of the indicated genes (B, C, D).

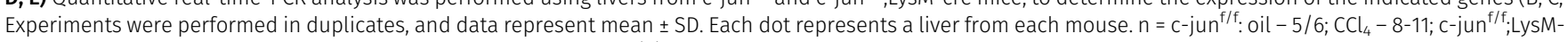
cre: oil $-3 / 4 ; \mathrm{CCl}_{4}-5-8$. Statistics was performed by unpaired $t$ test. (E) Whole liver lysates from the indicated groups of mice were used for immunoblotting analysis with the indicated antibodies as described above (E). (F) Compensation by macrophages for the lack of c-Jun function specifically in hepatocytes. Fibrotic signals activate c-Jun in hepatocytes and KCS, leading to production of cytokines and thus fibrosis (extent arbitrarily indicated by ++). In the absence of c-Jun in hepatocytes only, the KCS over-compensate for the deficiency, which results in overall elevated fibrosis (+++). However, in the absence of c-Jun in hepatocytes and KCs, or KCs alone, cytokine production is diminished, and results in reduced fibrosis (+ or ++).

Source data are available for this figure.

efficient expression of cytokine genes in hepatocytes as well as the $\mathrm{F} 4 / 80^{+}$macrophages, both of which contribute to liver fibrosis. However, absence of c-Jun in hepatocytes alone leads to a compensatory increase in the functions of $\mathrm{KCS}$, which therefore lead to enhanced fibrosis in the case of the $c-j u n^{f / f} ; A l b$-cre mice.

\section{c-jun deletion does not affect fibrosis resolution}

Given the significant role of c-Jun in mediating liver fibrosis through both the hepatocytes and KCs, we further evaluated if it is also involved in the resolution phase, after removal of the fibrotic stimuli. Initial analysis of c-jun expression, which is induced by $\mathrm{CCl}_{4}$ treatment, indicated that the expression was maintained over 8-wk posttreatment (Fig 6A). Similar results were obtained by immunoblot analysis despite the almost complete decrease in Acta2 expression, reflecting the resolution of fibrosis (Fig 6B). Hence, to evaluate if there is a causal role for c-Jun in the resolution process, fibrosis was induced by $\mathrm{CCl}_{4}$ treatment for $6 \mathrm{wk}$, and the livers were

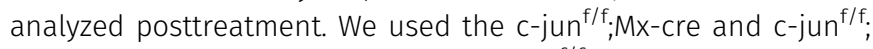
LysM-cre mice for analysis, as the $\mathrm{c}$-jun $^{\mathrm{f} / \mathrm{f}} ; \mathrm{Alb}$-cre mice already started developing nodules on the livers after $8 \mathrm{wk}$ of $\mathrm{CCl}_{4}$ treatment (Fig S4A), and hence, were not included in this study. c-jun ${ }^{\mathrm{f} / \mathrm{f}}$; $M x$-cre mice were injected with Poly-I:C 1 wk before the end of the $\mathrm{CCl}_{4}$ treatment to delete $c$-jun, which continued till the end of the 
A

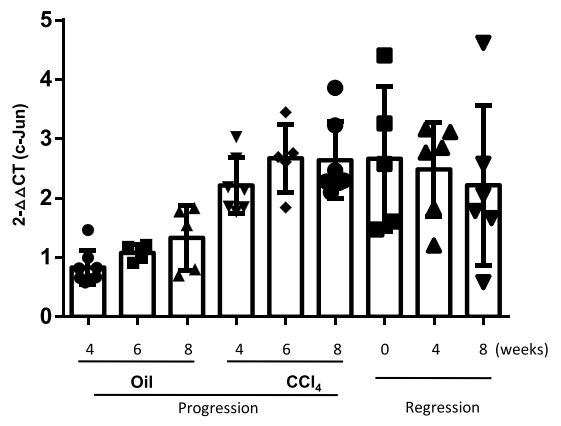

B

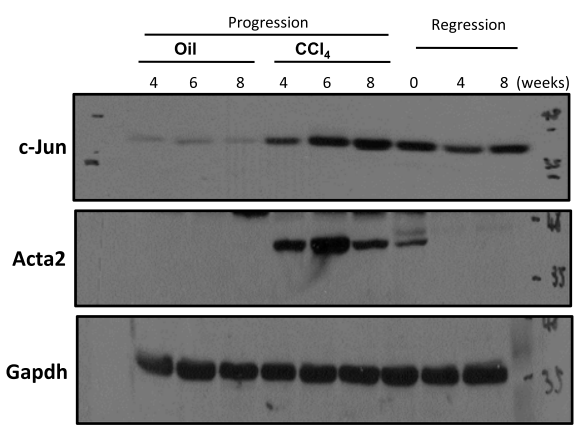

C

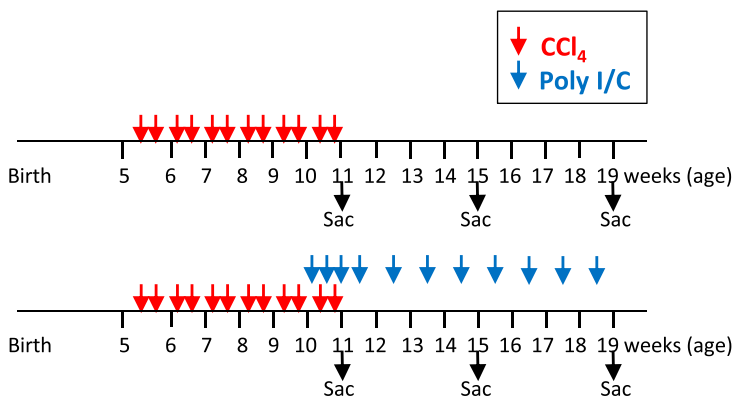

D

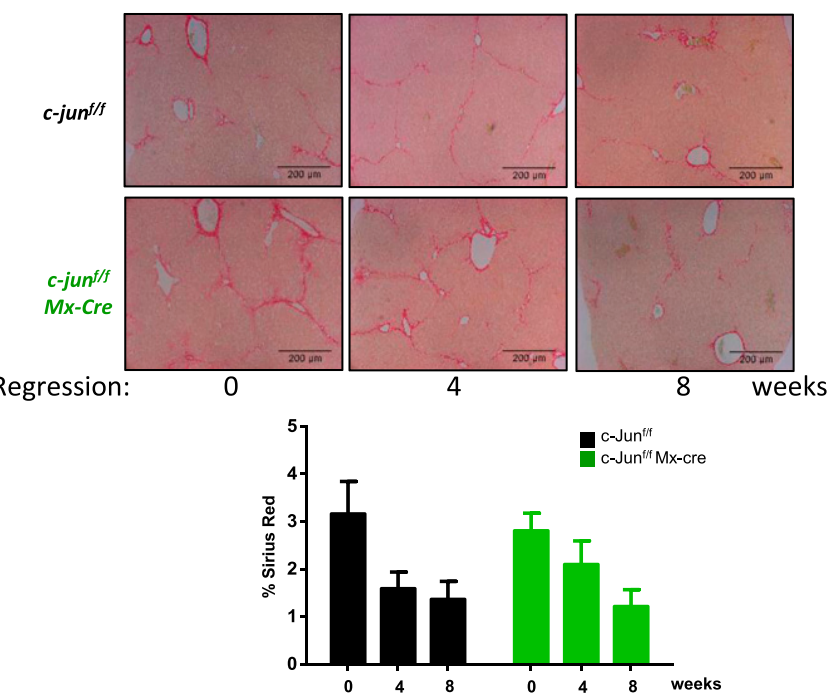

$\mathbf{E}$
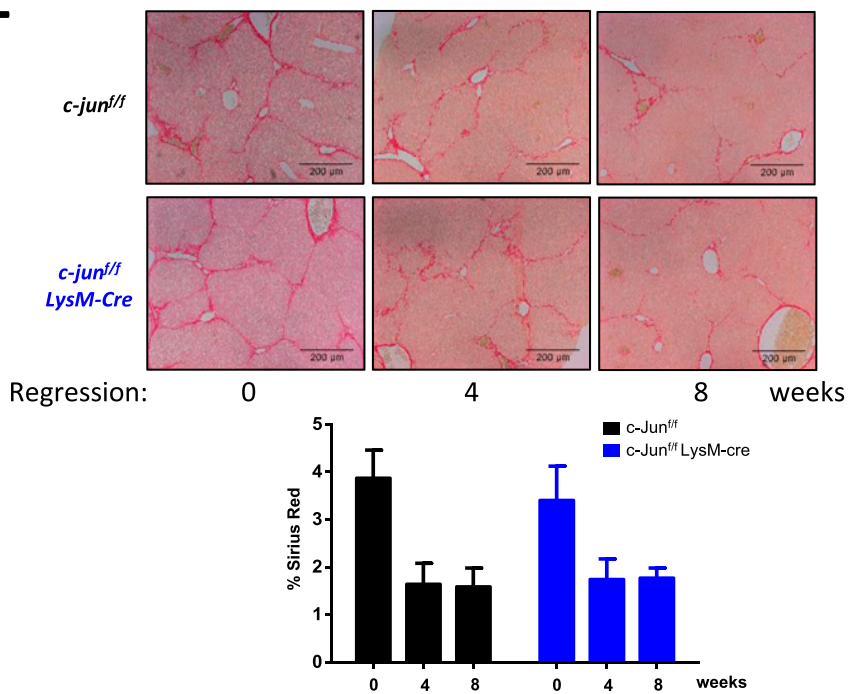

Figure 6. c-jun deletion in Kupffer cells alone or together with hepatocytes does not affect fibrosis resolution.

(A, B) Expression of c-jun was determined by quantitative real-time-PCR (A) and by immunoblotting (B) using whole livers from mice treated with oil or $\mathrm{CCl}_{4}$-tretment (progression), and for 8 wk posttreatment (regression). Experiments were performed in duplicates and data represent mean \pm SD. Each dot represents a liver from an individual mouse. $n=c-j u n^{f / f}:$ oil: $4 w k-8 ; 6 w k-4 ; 8 w k-5 ; c-j u n^{f / f}: c C l l_{4}: 4 w k-7 ; 6 w k-5 ; 8 w k-7 ; c-j u n^{f / f}$ Regression: 0 wk $-5 ; 4$ wk $-6 ; 8$ wk- 6 . Statistics was performed by unpaired $t$ test. (C) Schematic of fibrosis regression studies. Mice were either treated with oil or $\mathrm{CCl}_{4}$ twice per week for 6 wk to induce fibrosis. Poly I:C was injected as indicated to induce the expression of $\mathrm{Cre}$ in $\mathrm{Mx}$ - $\mathrm{Cre}$, from the last week of $\mathrm{CCl}_{4}$ injection till the end of the experimental period of 8 wk post- $\mathrm{CCl}_{4}$ treatment. Mice were harvested at either 0,4 , or $8 \mathrm{wk}$ from the cessation of $\mathrm{CCl}_{4}$ treatment. (D, E) Extent of liver fibrosis and damage over 8 wk post-CCl 4 treatment was determined by Sirius red staining (top panels), and representative pictures are shown (10x magnification). Quantification of fibrosis is based on analyzing 20 randomly chosen fields from four individual liver lobes per mouse (lower panels). Data represent mean +SD. $n=c-j u n^{f / f}: 0 w k-8 ; 4 w k-8 ; 8 w k-7 ; c-j u n^{f / f} ; M x-c r e: 0 w k-8 ; 4 w k-7 ; 8 w k-8 ; c-j u n^{f / f}: 0 w k-9$; $4 w k-8 ; 8 w k-10 ; c-j u n^{f / f} ;$ iLsM-cre: $0 w k-8 ; 4 w k-6 ; 8 w k-6$. Statistics was performed by two-way ANOVA.

Source data are available for this figure.

experiment (Figs $6 \mathrm{C}$ and S2E), and livers were harvested at 4 and 8 wk post- $\mathrm{CCl}_{4}$ treatment and analyzed. As shown in Fig 6D, deletion of c-jun did not markedly affect the fibrosis resolution process in the $\mathrm{c}$-jun ${ }^{\mathrm{f} / \mathrm{f}} ; \mathrm{Mx}$-cre mice (\% Sirius red staining of $\mathrm{CCl}_{4}$-treated livers: without or with $\mathrm{Mx}$-cre at the following time points [weeks] - 0: $3.160 \pm$ 0.684 versus $2.805 \pm 0.374 ; P=0.6294 ; 4: 1.588 \pm 0.353$ versus $2.098 \pm$ $0.496 ; P=0.2787 ; 8: 1.363 \pm 0.379$ versus $1.215 \pm 0.353 ; P=0.9880$ ). Consistently, whereas the molecular markers associated with fibrosis decreased over time, no significant differences were noted because of the absence of c-Jun, as shown for Acta2 and Vimentin (relative expression of $A c t a 2$ in livers of $\mathrm{CCl}_{4}$-treated regressed mice: without or with $\mathrm{Mx}$-cre at the following time points [weeks] - 0: $0.8850 \pm 0.3717$ versus $0.8735 \pm 0.2132 ; P=0.9999 ; 4: 0.8686 \pm 0.1878$ versus $0.6636 \pm$ $0.1108 ; P=0.8310 ; 8: 0.7665 \pm 0.1349$ versus $0.5015 \pm 0.1239 ; P=0.6452$; Vimentin $-0: 1.946 \pm 1.079$ versus $1.844 \pm 0.8240 ; P=0.9999 ; 4: 1.632 \pm$ 1.210 versus $1.027 \pm 0.2873 ; P=0.9243 ; 8: 1.071 \pm 0.4948$ versus $0.6627 \pm$ 


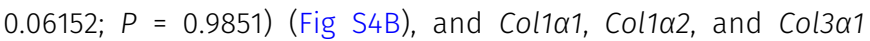
(relative expression of Col1a1 in livers of $\mathrm{CCl}_{4}$-treated regressed mice: without or with $\mathrm{Mx}$-cre at the following time points [weeks] $0: 6.066 \pm 1.557$ versus $2.998 \pm 1.823 ; P=0.049 ; 4: 1.127 \pm 0.6879$ versus $0.6947 \pm 0.06344 ; P=0.9931 ; 8: 0.7872 \pm 0.5212$ versus $0.2041 \pm 0.1185$; $P=0.9742$; Col1a2 $-0: 3.342 \pm 1.655$ versus $2.973 \pm 0.5303 ; P=0.9983$; 4: $1.733 \pm 1.222$ versus $1.456 \pm 0.3733 ; P=0.9992 ; 8: 1.037 \pm 0.7700$ versus $0.6706 \pm 0.2067 ; P=0.9983 ;$ Col3a1 $-0: 4.056 \pm 0.8880$ versus $3.568 \pm 1.020 ; P=0.9751 ; 4: 1.550 \pm 1.257$ versus $0.9002 \pm 0.4455 ; P=$ $0.9211 ; 8: 0.9227 \pm 0.6937$ versus $0.4520 \pm 0.08341 ; P=0.9787$ ) (Fig $\mathrm{S} 4 \mathrm{C})$. Moreover, the expression of the tissue inhibitor of metalloproteinases (TIMPS) 1 and 2 which are down-regulated during liver fibrosis resolution, as well as the matrix metellopeptidases (MMPs) 8 and 13 which are induced during fibrosis resolution (Giannandrea \& Parks, 2014) were not significantly altered because of the absence of c-Jun (relative expression of Timp1 in livers of $\mathrm{CCl}_{4}$-treated regressed mice: without or with $\mathrm{Mx}$-cre at the following time points [weeks] -- 0: $3.722 \pm 3.475$ versus $3.444 \pm$ $0.6431 ; P=0.9999 ; 4: 2.015 \pm 0.8716$ versus $2.842 \pm 1.661 ; P=0.9898 ; 8$ : $1.750 \pm 1.226$ versus $0.9351 \pm 0.3654 ; P=0.9904 ;$ Timp $2-0: 1.884 \pm$ 0.9538 versus $2.130 \pm 0.7394 ; P=0.9957 ; 4: 1.814 \pm 0.5711$ versus 1.443 $\pm 0.4122 ; P=0.9729 ; 8: 1.308 \pm 0.5455$ versus $0.5661 \pm 0.1434 ; P=$ $0.6839 ; \mathrm{mmp} 8-0: 0.7559 \pm 0.05902$ versus $0.8920 \pm 0.2225 ; P=$ $0.9991 ; 4: 0.9880 \pm 0.3799$ versus $1.177 \pm 0.4334 ; P=0.9933 ; 8: 1.229 \pm$ 0.4459 versus $1.438 \pm 0.499 ; P=0.9829 ; m m p 13-0: 0.5594 \pm 0.4620$ versus $0.4415 \pm 0.1429 ; P=0.9999 ; 4: 0.8231 \pm 0.7412$ versus $0.5764 \pm$ $0.3448 ; P=0.9972 ; 8: 2.048 \pm 1.091$ versus $2.889 \pm 1.211 ; P=0.8158$ ) (Fig S4D and E). Similar results were obtained using $\mathrm{C}-\mathrm{jun} \mathrm{n}^{\mathrm{f} / \mathrm{f}} ; \mathrm{LysM}$-cre mice for fibrosis resolution (\% Sirius red staining of $\mathrm{CCl}_{4}$-treated livers: without or with LysM-cre at the following time points [weeks] - 0: $3.869 \pm 0.589$ versus $3.401 \pm 0.722 ; P=0.4128 ; 4: 1.640 \pm$ 0.443 versus $1.741 \pm 0.433 ; P=0.9990 ; 8: 1.584 \pm 0.399$ versus $1.768 \pm$ $0.215 ; P=0.9820)$, and the expression of the molecular markers (relative expression of Acta2 in livers of $\mathrm{CCl}_{4}$-treated regressed mice: without or with LysM-cre at the following time points [weeks] $-0: 1.024 \pm 0.2616$ versus $0.785 \pm 0.04748 ; P=0.4489 ; 4: 0.8891 \pm$ 0.1436 versus $0.6433 \pm 0.1056 ; P=0.4197 ; 8: 0.8557 \pm 0.1970$ versus $0.5133 \pm 0.03898 ; P=0.1421$; Vimentin $-0: 1.235 \pm 0.3104$ versus 1.058 $\pm 0.2877 ; P=0.9803 ; 4: 0.9683 \pm 0.4521$ versus $0.7856 \pm 0.4128 ; P=$ $0.9777 ; 8: 0.6567 \pm 0.1874$ versus $0.4483 \pm 0.1257 ; P=0.9613$; Col1a1 -0 : $3.293 \pm 0.9085$ versus $2.706 \pm 1.046 ; P=0.9244 ; 4: 0.6908 \pm 0.4724$ versus $1.017 \pm 0.7698 ; P=0.9896 ; 8: 0.7012 \pm 0.2114$ versus $0.5485 \pm$ $0.5340 ; P=0.9997$; Col1a2 $-0: 1.895 \pm 1.059$ versus $2.194 \pm 1.508 ; P=$ $0.9988 ; 4: 1.185 \pm 1.044$ versus $1.175 \pm 0.8429 ; P=0.9999 ; 8: 0.8234 \pm$ 0.2167 versus $0.7506 \pm 0.3485 ; P=0.9999 ;$ Col3a1 $-0: 2.069 \pm 0.8903$ versus $2.912 \pm 0.9697 ; P=0.5828 ; 4: 0.7596 \pm 0.4374$ versus $0.7815 \pm$ $0.4024 ; P=0.9999 ; 8: 0.7119 \pm 0.2856$ versus $0.3862 \pm 0.1129 ; P=$ 0.9760; Timp1 $-0: 1.934 \pm 1.347$ versus $2.371 \pm 1.564 ; P=0.9984 ; 4: 1.159$ \pm 1.209 versus $1.995 \pm 2.295 ; P=0.9704 ; 8: 0.7730 \pm 0.2090$ versus 1.209 $\pm 0.1380 ; P=0.9984 ;$ Timp $2-0: 0.9842 \pm 0.1702$ versus $0.9948 \pm$ $0.09253 ; P=0.9999 ; 4: 0.8715 \pm 0.4498$ versus $0.8904 \pm 0.5435 ; P=$ $0.9999 ; 8: 0.6796 \pm 0.6441$ versus $0.6415 \pm 0.2215 ; P=0.9999 ; \mathrm{mmp} 8-$ $0: 0.7377 \pm 0.08479$ versus $0.3740 \pm 0.2410 ; P=0.7365 ; 4: 0.6134 \pm$ 0.1370 versus $0.9259 \pm 0.2685 ; P=0.6974 ; 8: 1.343 \pm 0.4060$ versus $1.190 \pm 0.2151 ; P=0.9848 ; m m p 13-0: 0.3456 \pm 0.1746$ versus $0.2246 \pm$ $0.1359 ; P=0.9999 ; 4: 1.057 \pm 0.5640$ versus $0.7145 \pm 0.1054 ; P=0.9948$; $8: 2.840 \pm 1.767$ versus $3.000 \pm 0.7627 ; P=0.9999$ ) (Figs $6 \mathrm{E}$ and $4 \mathrm{~B}-\mathrm{E}$ ).
These results together indicate that c-Jun is not required for the fibrosis resolution process.

\section{Discussion}

We have in this study investigated the interplay between cell types in orchestrating the liver fibrotic process, using c-Jun deficiency in hepatocytes and macrophages as a model. c-Jun, being a critical transcription factor implicated in the biology of hepatocytes (Hilberg et al, 1993; Behrens et al, 2002; Eferl et al, 2003), also plays crucial roles in regulating the functions of macrophages (Hannemann et al, 2017). In both cases, c-Jun is necessary for activation of appropriate target genes which regulate the proper functioning of the cells. Using c-Jun deficiency as a means to functionally alter these cell types, we show that a coordinated response between hepatocytes and macrophages is necessary to regulate the liver fibrotic process (Fig 5F).

We demonstrate here that c-Jun acts as a pro-fibrogenic factor in both hepatocytes and $\mathrm{KCs}$ during liver fibrosis mediated by $\mathrm{CCl}_{4}$. Yet, absence of c-Jun in hepatocytes alone or in combination with $\mathrm{KCS}$ results in opposite outcomes. Isolated hepatocytes from both cohorts of mice exhibit a defect in cytokine gene expression, suggestive of a non-cell autonomous mechanism that contributes to enhanced fibrosis when c-Jun is deleted in hepatocytes alone. This is attributable to elevated macrophage numbers in the livers of the $c$-jun ${ }^{f / f} ; A l b$-cre mice. Consistently, depletion of macrophages in these mice led to the reversal of the phenotype, confirming a compensatory effect of the macrophages during liver fibrosis when hepatocyte functions are specifically affected. These findings further demonstrate that an orchestrated coordination between hepatocytes and macrophages is crucial in regulating the fibrotic process. On the other hand, deletion of c-jun in KCs also led to decreased fibrosis, reiterating a pro-fibrogenic role for $\mathrm{KCs}$. However, deletion of $c$-jun in $\mathrm{KCS}$, which are a minor population in the liver compared with hepatocytes, did not appear to lead to any significant compensation by hepatocytes. In this regard, it is interesting to note that the magnitude of decrease in the expression of cytokine genes was significantly greater than the degree of fibrosis as ascertained by Sirius red staining when c-jun was deleted in KCS (Fig 5A, B, and E), indicating that the impact of defects in the minor KC population on overall liver fibrosis is relatively minimal. Not surprisingly, deletion of c-jun in both these cell types led to reduced fibrosis, consistent with their profibrogenic role.

A noteworthy point is that we had used LysM-cre strain for deletion of $\mathrm{c}$-jun in the KCs. LysM-cre mice have been extensively used for deletion in macrophages, and in particular, KCs in liver disease contexts (Greenhalgh et al, 2015; Beattie et al, 2016; Shi et al, 2018; Puchalska et al, 2019). However, they also delete in granulocytes (Shi et al, 2018), and the role of c-Jun in granulocytes have yet to be explored. Nevertheless, our data using chemical depletion

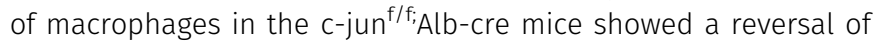
the fibrotic phenotype, corroborating with the LysM-cre data and alludes strongly to a role for c-Jun in the KCs in contributing to liver fibrosis. 
Genotyping was performed using the following primers, with the expected size of PCR products are indicated in the table below:

\begin{tabular}{lll} 
Primers & Sequence & Expected PCR size (bp) \\
\hline c-Jun lox5 (For) & CTCATACCAGTTCGCACAGGC & 350 \\
\hline c-Jun lox6 (Rev 1) & CGCTAGCACTCACGTTGGTAG & $4,000 / 600$ \\
\hline c-Jun flox2 (Rev 2) & CAGGGCGTTGTGTCACTGAGCT & 324 \\
\hline Cre internal Ctrl (For) & CTAGGCCACAGAATTGAAAGATCT & 500 \\
\hline Cre internal Ctrl (Rev) & GTAGGTGGAAATTCTAGCATCATCC & \\
\hline Cre transgene (For) & TCCAATTTACTGACCGTACACCAA & \\
\hline Cre transgene (Rev) & CCTGATCCTGGCAATTTCGGCTA & \\
\hline
\end{tabular}

The phenomenon of compensatory increase in macrophages in the liver appears to be conserved with respect to liver damage. Absence of Egfr signaling in hepatocytes alone led to increased HCC formation induced by diethylnitrosamine/phenobarbital because of the compensatory increase in Egfr signaling in KCs (Lanaya et al, 2014). By contrast, HCC rates were reduced when Egfr was deleted in both hepatocytes and $\mathrm{KCS}$ (using the Mx-cre mice), or in $\mathrm{KC}$ cells alone (LysM-cre). Similarly, deletion of IKK $\beta$ in hepatocytes alone led to increased hepatocarcinogenesis induced by dietheylnitrosamine (DEN), which was reversed upon IKK $\beta$ deletion using $M x-$ cre mice (Maeda et al, 2005). Altogether, our data are consistent with previous studies of liver carcinogenesis and supports the notion that an orchestrated cross-talk between KCS and hepatocytes is required to regulate the liver fibrotic process.

A recent study that was published while our manuscript was under review also explored the role of c-Jun in non-alcoholic steotohepatitis (NASH) induced by methionine-choline-deficient diet (MCDD), which leads to liver fibrosis (Schulien et al, 2019). The conclusions of that study were also broadly similar to our work although with a different mechanistic basis. In essence, the MCDD-induced fibrosis and NASH also affects adipogenesis, unlike the $\mathrm{CCl}_{4}$-induced liver fibrosis. This is reflected in the different causal mechanisms that are predominant in these models. In the MCDD model, c-Jun has been suggested to regulate the expression of Opn and its receptor CD44 in non-parenchymal cells through the ductular reaction, leading to increased fibrosis in the liver-specific knockout. There was, however, no increase in F4/80+ cells in the Alb-cre model (rather, there was a decrease). By contrast, we see a significant increase in the numbers of F $4 / 80$ + cells in the Alb-cre knockout model, which has led us to deplete macrophages chemically and delete c-jun in macrophages using the LysM-Cre model. Furthermore, the MCDD model leads to increased cell death in the livers of the liver-specific knockout mice, which was reduced in the MX-cre model. However, in our $\mathrm{CCl}_{4}$ model, we do not see any difference in cell death in the $M x$-cre model, again highlighting the mechanistic differences attributable to c-Jun deficiency in the different models. Altogether, although both studies have broadly come to similar conclusions, the various differences highlighted above complement both studies which together demonstrate the similarities in the broad principles of liver fibrosis regulation by c-Jun.

Our study has further explored the role of c-Jun in the fibrosis resolution process, which revealed that c-Jun expression was maintained significantly in the liver even 8 wk post-removal of the fibrotic stimulation. However, deletion of c-jun during this phase had no marked effects on the resolution rate or on the changes in any of the molecular markers, indicating that c-Jun expression is not critical for the recovery phase when the fibrotic stimuli is removed. This prompts us to speculate that the levels of c-Jun might be maintained as a provisionary measure for the rapid re-activation of the fibrotic process in the event that the fibrotic stimuli reemerges, an hypothesis that requires future investigation.

Many molecular pathways, including several AP-1 pathway members have been shown to be active in and causal to fibrosis (Smart et al, 2006; Omenetti \& Diehl, 2011; Wernig et al, 2017), and therapeutic interventions to target them are being explored. One caveat the current study highlights is the complexity of the systems and the interplay of cell types within an organ that is critical in controlling the collective eventual outcome, which would likely be similar for other signaling modules. Hence, it is important to understand the functions of the major signaling pathways in multiple tissues and cell types within them, before embarking on efforts to target them. Thus, any molecular therapeutic intervention requires judicious interrogation so as not to perturb the balance between cell types.

In summary, our study demonstrates yet another level of complexity of cellular signaling, using c-Jun as a model, in maintaining liver homeostasis during fibrosis. This work highlights the tight regulation that is maintained among cell types, which dictates the extent of fibrosis. It is likely that such a paradigm might be operative in other organs, and in other pathological conditions such as those that lead to wound healing. Hence, careful consideration ought to be given when using molecularly driven drugs.

\section{Materials and Methods}

\section{Mouse breeding, induction of fibrosis, and perfusion}

Mouse strains used in the study are as follows: $c$-jun ${ }^{\mathrm{f} / \mathrm{f}}$ (Behrens et al, 2002); Alb-creER; LysM-cre; and Mx-cre (Lanaya et al, 2014). All mice were bred, housed, and used for experiments with the approval and in accordance with the guidelines of the SingHealth's Animal Care and Use Committee. 
6-wk-old mice were treated twice a week with olive oil or carbon tetrachloride $\left(\mathrm{CCl}_{4}\right)(1 \mu \mathrm{l}$ per gram body weight [gbw] diluted in olive oil) by i.p. injection for 8 wk to induce fibrosis. Alb-creER (i.e., Alb) mice were in addition treated with tamoxifen ( $1 \mathrm{mg}$ per mouse) by oral gavage to activate the Cre-recombinase (as per schedule shown in Fig 1B). Mice expressing Mx-cre were administered with Poly I:C (13 $\mu \mathrm{g} / \mathrm{gbw})$ by i.p. injection to induce Cre expression $1 \mathrm{wk}$ before $\mathrm{CCl}_{4}$ injection. $10 \mu \mathrm{g} / \mathrm{gbw}$ of gadolinium chloride $\left(\mathrm{GdCl}_{3}\right)$ or PBS was injected i.v. once a week for 8 wk during the course of $\mathrm{CCl}_{4}$ injection, in the indicated studies.

For regression studies, 6-wk-old mice were treated twice a week with olive oil or $\mathrm{CCl}_{4}$ as described for 6 wk to induce fibrosis. Mice expressing Mx-cre were administered with Poly I:C (13 $\mu \mathrm{g} / \mathrm{gbw})$ by i.p. injection to induce Cre expression $5 \mathrm{wk}$ after the start of the $\mathrm{CCl}_{4}$ injection. Mice were kept for an additional of 4 or 8 wk after the last $\mathrm{CCl}_{4}$ injection and analyzed thereafter.

All mice were euthanized $72 \mathrm{~h}$ after the last $\mathrm{CCl}_{4}$ injection and their livers were excised, frozen, or formalin-fixed for further analysis.

For quantification on the efficiency of c-jun deletion, image J was used to measure the intensity of bands. These values were then converted as a percentage of the floxxed band over the total floxxed and unfloxxed bands.

For perfusion and collection of cells, mice were anaesthetized by i.p. injection of $5 \mu \mathrm{g} / \mathrm{gbw}$ Diazepam and $50 \mu \mathrm{g} / \mathrm{gbw}$ Ketamine. Mice were then perfused from inferior vena cava and out from the hepatic portal vein. $3.7 \mathrm{U}$ of collagenase $\mathrm{D}$ was used to flush through the liver before ex-vivo digestion using the same solution for $5 \mathrm{~min}$. Cells were then passed through a $70 \mu \mathrm{M}$ strainer, and centrifuged at $50 \mathrm{~g}$ for $10 \mathrm{~min}$ to isolate out purified hepatocytes (Severgnini et al, 2012).

\section{Quantitative gene expression assay}

Total RNA was prepared and used to synthesize cDNA using SuperScript II reverse transcriptase, as described (Subramanian et al, 2015). qRT-PCR was performed using gene-specific primers (Table S1) and QuantiFast SYBR Green PCR Kit in Rotor-Gene Q real-time PCR machine (QIAGEN) and ViiA7 real-time PCR system (Thermo Fisher Scientific) according to manufacturer's instructions, and relative gene expression was normalized with Gapdh expression. $2-\Delta \Delta$ Ct was used to compare the transcriptional differences. Cycle threshold $(\mathrm{Ct})$ values of various genes were plotted against $\mathrm{Ct}$ values of Gapdh to determine a ratio between different treatment groups.

\section{Immunoblot analysis}

Small pieces of liver tissues were lysed and $\sim 80 \mu \mathrm{g}$ of proteins were used and analyzed by immunoblot. Antibodies against c-Jun (9165; CST), Acta-2 (M0851; Dako), Desmin (14026; Santa Cruz), Vimentin (5741; CST), TGF- $\beta 1$ (130348; Santa Cruz), TNF- $\alpha$ (52746; Santa Cruz), Gapdh (25778; Santa Cruz), p-JNK (9255; CST), and t-JNK (7345; Santa Cruz) were used in this work. Multiple gels were run with the same set of lysates from each cohort of mice to be probed with the various antibodies, with one or two blots being used for assessment of loading using the anti-Gapdh antibody.

\section{Histological and related analysis}

Liver tissues (all lobes) were fixed in 10\% formalin overnight, and then dehydrated and embedded in paraffin blocks, which were sectioned at a thickness of $5 \mu \mathrm{m}$. For Sirius red staining, sections were stained with hematoxylin (Weigert's) for 8 min, washed with running tap water for 10 min, followed by incubation with $0.1 \%$ (wt/vol) Sirius red diluted in picric acid solution for $1 \mathrm{~h}$. The slides were then rinsed in two quick changes of $0.5 \%$ ( $\mathrm{vol} / \mathrm{vol}$ ) acetic acid to remove unbound dye. For immunostaining, sections were first heated in sodium citrate buffer to retrieve antigen. Sections were then blocked in 10\% FBS blocking buffer followed by incubation with the following primary antibodies: anti-c-Jun (60A8; Cell Signaling), anti-F4/80 (SP115; Thermo Fisher Scientific), and anti-Ki67 (15580; Abcam), before quenching endogenous peroxidase using 3\% hydrogen peroxide. TUNEL staining was performed as per manufacturer's recommendations using the apoptosis detection kit (206386; Abcam). To quantify staining, 20 randomly taken images of $10 \times$ fields per section were evaluated by Meta Imaging Series (Molecular Devices) software.

A small portion of the liver was homogenized in ice cold $\mathrm{NaCl}$ buffer. The supernatant was collected, normalized before subjected to AST/ALT measurements using an AST/ALT Assay kit (Nanjing Jiancheng Bioengineering Institute) according to the supplier's protocol.

\section{Statistical analysis}

All data are presented as mean \pm SD. The results were analyzed by unpaired $t$ test, ANOVA or by two-way ANOVA, as appropriate. Statistical calculation was performed using GraphPad Prism software (5.0). The animal numbers used for each experiment are indicated in each of the figure legends. $P$-values less than 0.05 were considered to be statistically significant.

\section{Supplementary Information}

Supplementary Information is available at https://doi.org/10.26508/lsa. 202000803.

\section{Acknowledgements}

The authors thank the following for the generous gift of the mouse strains, cell lines, and reagents: Axel Behrens (c-Jun f/f mice), Erwin Wagner (Alb-cre mice), and Zhao-Qi Wang (Mx-cre mice). We also thank Philipp Kaldis, Matias Jose Caldez, and Noemi Kathleen and Marcelle Vanhul (IMCB, Singapore) for guiding us through the liver perfusion protocol; Ralph Bunte (Duke-NUS, Singapore) for advice of histopathology; and Anna-Mae Diehl (Duke University, USA) for advice and support on the liver fibrosis studies. This work was supported by grants from National Medical Research Council of Singapore (NMRC) (to K Sabapathy) (NMRC/CBRG/0061/2014).

\section{Author Contributions}

M Xie: data curation, software, formal analysis, validation, investigation, visualization, methodology, and writing-review and editing 
RH Chia: data curation, software, formal analysis, validation, investigation, visualization, methodology, and writing-review and editing.

D Li: data curation, formal analysis, validation, investigation, visualization, methodology, and writing-review and editing.

FX Teo: data curation, validation, investigation, visualization, and methodology.

C Krueger: data curation, validation, investigation, visualization, and methodology.

K Sabapathy: conceptualization, resources, formal analysis, supervision, funding acquisition, investigation, visualization, methodology, project administration, and writing-original draft, review, and editing.

\section{Conflict of Interest Statement}

The authors declare that they have no conflict of interest

\section{References}

Beattie L, Sawtell A, Mann J, Frame TC, Teal B, de Labastida Rivera F, Brown M, Walwyn-Brown K, Moore JWJ, MacDonald S, et al (2016) Bone marrowderived and resident liver macrophages display unique transcriptomic signatures but similar biological functions. J Hepatol 65: 758-768. doi:10.1016/j.jhep.2016.05.037

Behrens A, Sibilia M, David JP, Möhle-Steinlein U, Tronche F, Schütz G, Wagner EF (2002) Impaired postnatal hepatocyte proliferation and liver regeneration in mice lacking c-jun in the liver. EMBO J 21: 1782-1790. doi:10.1093/emboj/21.7.1782

Cubero FJ, Zoubek ME, Hu W, Peng J, Zhao G, Nevzorova YA, Al Masaoudi M, Bechmann LP, Boekschoten MV, Muller M, et al (2016) Combined activities of JNK1 and JNK2 in hepatocytes protect against toxic liver injury. Gastroenterology 150: 968-981. doi:10.1053/j.gastro.2015.12.019

Eferl R, Ricci R, Kenner L, Zenz R, David JP, Rath M, Wagner EF (2003) Liver tumor development: c-Jun antagonizes the proapoptotic activity of p53. Cell 112: 181-192. doi:10.1016/s0092-8674(03)00042-4

Eferl R, Wagner EF (2003) AP-1: A double-edged sword in tumorigenesis. Nat Rev 3: 859-868. doi:10.1038/nrc1209

Giannandrea M, Parks WC (2014) Diverse fucntions of matrix metalloproteinases during fibrosis. Dis Model Mech 7: 193-203. doi:10.1242/dmm.012062

Greenhalgh SN, Conroy KP, Henderson NC (2015) Cre-activity in the liver: Transgenic approaches to targeting hepatic non parenchymal cells Hepatology 61: 2091-2099. doi:10.1002/hep.27606

Guillot A, Tacke F (2019) Liver macrophages: Old dogmas and new insights. Hepatol Commun 3: 730-743. doi:10.1002/hep4.1356

Hannemann N, Jordan J, Paul S, Reid S, Baenkler HW, Sonnewald S, Bäuerle T, Vera J, Schett G, Bozec A (2017) The AP-1 transcription factor c-Jun promotes arthritis by regulating cyclooxygenase-2 and arginase- 1 expression in macrophages. J Immunol 198: 3605-3614. doi:10.4049/ jimmunol.1601330

Hilberg F, Aguzzi A, Howells N, Wagner EF (1993) c-Jun is essential for normal mouse development and hepatogenesis. Nature 365: 179-181. doi:10.1038/365179a0

Ju C, Tacke F (2016) Hepatic macrophages in homeostasis and liver diseases: From pathogenesis to novel therapeutic strategies. Cell Mol Immunol 13: 316-327. doi:10.1038/cmi.2015.104

Kodama Y, Kisseleva T, Iwaisako K, Miura K, Taura K, De Minicis S, Osterreicher CH, Schnabl B, Seki E, Brenner DA (2009) C-Jun N-terminal kinase-1 from hematopoietic cells mediates progression from hepatic steatosis to steatohepatitis and fibrosis in mice. Gastroenterology 137: 1467-1477. doi:10.1053/j.gastro.2009.06.045

Koyama Y, Brenner DA (2017) Liver inflammation and fibrosis. J Clin Invest 127 55-64. doi:10.1172/jci88881

Lanaya H, Natarajan A, Komposch K, Li L, Amberg N, Chen L, Wculek SK, Hammer M, Zenz R, Peck-Radosavljevic M, et al (2014) EGFR has a tumour-promoting role in liver macrophages during hepatocellular carcinoma formation. Nat Cell Biol 16: 972-981. doi:10.1038/ncb3031

Machado MV, Diehl AM (2018) Hedgehog signalling in liver pathophysiology. J Hepatol 68: 550-562. doi:10.1016/j.jhep.2017.10.017

Maeda S, Kamata H, Luo JL, Leffert H, Karin M (2005) IKKbeta couples hepatocyte death to cytokine-driven compensatory proliferation that promotes chemical hepatocarcinogenesis. Cell 121: 977-990. doi:10.1016/j.cell.2005.04.014

Michelotti GA, Machado MV, Diehl AM (2013) NAFLD, NASH and liver cancer. Nat Rev Gastroenterol Hepatol 10: 656-665. doi:10.1038/ nrgastro.2013.183

Moles A, Murphy L, Wilson CL, Chakraborty JB, Fox C, Park EJ, Mann J, Oakley F, Howarth R, Brain J, et al (2014) A TLR2/S100A9/CXCL-2 signaling network is necessary for neutrophil recruitment in acute and chronic liver injury in the mouse. J Hepatol 60: 782-791. doi:10.1016/ j.jhep.2013.12.005

Omenetti A, Diehl AM (2011) Hedgehog signaling in the liver. J Hepatol 54: 366-373. doi:10.1016/j.jhep.2010.10.003

Puchalska P, Martin SE, Huang X, Lengfeld JE, Daniel B, Graham MJ, Han X, Nagy L, Patti GJ, Crawford PA (2019) Hepatocyte-macrophage acetoacetate shuttle protects against tissue fibrosis. Cell Metab 29: 383-398. doi:10.1016/j.cmet.2018.10.015

Ramachandran P, Iredale JP, Fallowfield JA (2015) Resolution of liver fibrosis: Basic mechanisms and clinical relevance. Semin Liver Dis 35: 119-131. doi:10.1055/s-0035-1550057

Severgnini M, Sherman J, Sehgal A, Jayaprakash NK, Aubin J, Wang G, Zhang L, Peng CG, Yucius K, Butler J, et al (2012) A rapid two-step method for isolation of functional primary mouse hepatocytes: Cell characterization and asialoglycoprotein receptor based assay development. Cytotechnology 64: 187-195. doi:10.1007/s10616-0119407-0

Schulien I, Hockenjos B, Schmitt-Graeff A, Perdekamp MG, Follo M, Thimme R, Hasselblatt P (2019) The transcription factor c-Jun/AP-1 promotes liver fibrosis during non-alcoholic steatohepatitis by regulating Osteopontin expression. Cell Death Differ 26: 1688-1699. doi:10.1038/ s41418-018-0239-8

Seki E, Brenner DA, Karin M (2012) A liver full of JNK: Signaling in regulation of cell function and disease pathogenesis, and clinical approaches. Gastroenterology 43: 307-320. doi:10.1053/ j.gastro.2012.06.004

Shaulian E (2010) AP-1-The Jun proteins: Oncogenes or tumor suppressors in disguise? Cell Signal 22: 894-899. doi:10.1016/ j.cellsig.2009.12.008

Shi J, Hua L, Harmer D, Li P, Ren G (2018) Cre driver mice targeting macrophages. Methods Mol Biol 1784: 263-275. doi:10.1007/978-1 4939-7837-3_24

Smart DE, Green K, Oakley F, Weitzman JB, Yaniv M, Reynolds G, Mann J, Millward-Sadler H, Mann DA (2006) JunD is a profibrogenic transcription factor regulated by Jun $\mathrm{N}$-terminal kinase-independent phosphorylation. Hepatology 44: 1432-1440. doi:10.1002/hep.21436

Subramanian D, Bunjobpol W, Sabapathy K (2015) Interplay between TAp73 protein and selected activator protein-1 (AP-1) family members promotes AP-1 target gene activation and cellular growth. J Biol Chem 290: 18636-18649. doi:10.1074/jbc.m115.636548 
Sun B, Karin M (2008) NF-kappaB signaling, liver disease and hepatoprotective agents. Oncogene 27: 6228-6244. doi:10.1038/ onc.2008.300

Trautwein C, Friedman SL, Schuppan D, Pinzani M (2015) Hepatic fibrosis: Concept to treatment. J Hepatol 62: S15-S24. doi:10.1016/ j.jhep.2015.02.039

Wernig G, Chen SY, Cui L, Van NC, Tsai JM, Kambham N, Vogel H, Natkunam Y, Gilliland DG, Nolan G, et al (2017) Unifying mechanism for different fibrotic diseases. Proc Natl Acad Sci U S A 114: 4757-4762. doi:10.1073/ pnas. 1621375114

Zhao G, Hatting M, Nevzorova YA, Peng J, Hu W, Boekschoten MV, Roskams T, Muller M, Gassler N, Liedtke C, et al (2014) Jnk1 in murine hepatic stellate cells is a crucial mediator of liver fibrogenesis. Gut 63: 1159-1172. doi:10.1136/gutjnl-2013-305507

Zhu R, Guo W, Fang H, Cao S, Yan B, Chen S, Zhang K, Zhang S (2018) Kupffer cell depletion by gadolinium chloride aggravates liver injury after brain death in rats. Mol Med Rep 17: 6357-6362. doi:10.3892/ mmr.2018.8646

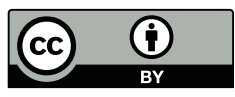

License: This article is available under a Creative Commons License (Attribution 4.0 International, as described at https://creativecommons.org/ licenses/by/4.0/). 